\title{
THE PATTERN OF INTERHEMISPHERIC CONNECTIONS AND ITS RELATIONSHIP TO EXTRASTRIATE VISUAL AREAS IN THE MACAQUE MONKEY ${ }^{1}$
}

\author{
DAVID C. VAN ESSEN, ${ }^{2}$ WILLIAM T. NEWSOME, ${ }^{3}$ AND JOHN L. BIXBY ${ }^{4}$ \\ Division of Biology, California Institute of Technology, Pasadena, California 91125
}

Received August 24, 1981; Revised November 12, 1981; Accepted November 12, 1981

\begin{abstract}
The distribution of interhemispheric connections was studied in extrastriate visual cortex of the macaque monkey. Callosal fiber terminations were identified by staining for anterograde degeneration following transection of the splenium of the corpus callosum. Retrogradely labeled cell bodies of callosal projection neurons were identified histochemically following application of horseradish peroxidase to the cut surface of the callosum. Results were displayed on unfolded, two-dimensional representations of the cortex, which permitted spatial and topological relationships between callosal recipient and callosal free cortical regions to be discerned readily.

The overall pattern of callosal inputs to visual cortex can be subdivided into nine callosal recipient zones which surround seven callosal free regions in the occipital, temporal, and parietal lobes. This pattern provides reliable and useful landmarks for identifying the borders of at least five topographically organized extrastriate visual areas. There is a pronounced dorsoventral asymmetry in callosal projections, not recognized in previous studies, which reflects an asymmetry in the organization of visual areas in dorsal versus ventral halves of the occipital lobe.

The pattern of callosal fiber terminations is mirrored by a very similar distribution of callosal projection neurons. There are significant regional differences in the laminar distribution of callosal projection cells, and these differences may reflect functionally distinct cortical subdivisions.

A considerable degree of individual variability was found in the relationship of callosal connections to gyral and sulcal landmarks as well as in the fine structure of individual callosal recipient strips, suggesting that each animal has a unique "callosal fingerprint." These findings emphasize the usefulness of the callosal pathway in elucidating the functional organization of extrastriate visual cortex.
\end{abstract}

The task of determining the number and organization of visual areas in the mammalian cerebral cortex has proven to be a formidable one. Nonetheless, substantial progress has been made on this problem over the past several decades. One of the important generalizations that has emerged from studies on a variety of higher mammals is that visually responsive cortex occupies a significantly larger portion of the hemisphere than pre-

\footnotetext{
${ }^{1}$ We thank A. Burkhalter, H. Gordon, C. Hamilton, J. Maunsell, and J. Allman for valuable criticisms of the manuscript, C. Shotwell and P. Knudsen for excellent technical assistance, and P. Brown for typing the manuscript. This work was supported by National Institutes of Health Grant RO1 EY02091, the Alfred P. Sloan Foundation, and the Pew Memorial Trust.

${ }^{2}$ To whom correspondence should be addressed.

${ }^{3}$ Present address: Laboratory of Sensorimotor Research, National Eye Institute, Bethesda, MD 20205.

${ }^{4}$ Present address: Department of Biology B-022, University of California, San Diego, La Jolla, CA 92093.
}

viously realized. Another is that visual cortex can be subdivided into a large number of distinct visual areas, not just the three areas envisioned by many classical anatomists (see Van Essen, 1979, for a review). Most of the known visual areas contain orderly representations of all or part of the contralateral half of the visual field. The analysis of visual topography has played a key role in establishing the existence and location of these areas. However, unambiguous identification of each area as a well defined entity is often not a straightforward matter. The most convincing assignments generally have been ones in which there is agreement among several independent approaches, including the analysis of topographic organization, architecture, connections, and functional properties of different cortical regions.

Of the various physiological and anatomical methods that have been used to determine the topographic organization of the cortex, one that has proven particularly useful in studies on the visual system is the analysis of 
interhemispheric connections. This approach is based on the finding that, over much of visual cortex, callosal fibers terminate preferentially in regions representing the vertical midline of the visual field (e.g., Choudhury et al., 1965; Hubel and Wiesel, 1965; Berlucchi, 1972; Sanides, 1978; Zeki, 1970; Van Essen and Zeki, 1978; Newsome and Allman, 1980). Since the vertical meridian is, in many instances, represented along the borders between visual areas, the overall pattern of callosal projections provides an anatomical reference system useful for demarcating the boundaries of several different areas in a single hemisphere. In the macaque monkey, such a reference system is especially valuable because well defined architectonic criteria for identifying borders have been lacking for most visual areas and because the complexity of the cortical convolutions make it difficult to map visual topography in great detail.

Previous studies in the macaque have demonstrated that callosal projections to visual cortex are arranged in a complex, highly intricate pattern whose major features are relatively consistent from one individual to another (Myers, 1962; Cragg, 1969; Zeki, 1969, 1970; Karol and Pandya, 1971; Pandya et al., 1971). Adequately detailed reconstructions of this pattern were not possible, however, until a technique was developed for constructing accurate two-dimensional maps of highly convoluted regions of cortex. In the original application of this technique, the distribution of interhemispheric connections was mapped in the dorsal half of the macaque occipital lobe (Van Essen and Zeki, 1978). This study demonstrated several unexpected features concerning the pattern of callosal projections to extrastriate visual cortex and revealed the presence of a previously unidentified visual area, V3A.

The procedure for constructing two-dimensional maps has been refined recently to the extent that the entire hemisphere can be represented accurately and consistently on a single cortical map (Van Essen and Maunsell, 1980). 'The primary objective of the present study was to take advantage of this improved technique in order to obtain an accurate and complete map of callosal inputs to visual cortex in the macaque. The results demonstrate a marked dorsoventral asymmetry in the pattern of interhemispheric connections which was not recognized in previous studies. This asymmetry, in turn, reflects important differences in the organization of visual areas in dorsal and ventral parts of the hemisphere which have been revealed by the tracing of intrahemispheric cortical connections. The second objective was to determine whether there is any consistent relationship between the local irregularities known to exist in the pattern of callosal inputs and the disorderliness in topographic organization that has been demonstrated in several extrastriate areas (Zeki, 1971, 1978d; Van Essen and Zeki, 1978; Van Essen et al., 1981). The third objective was to determine the distribution of the cells of origin of the callosal pathway. In particular, it was of interest to know how closely callosal projection neurons are restricted to regions of cortex which themselves receive inputs from the opposite hemisphere. An abstract of some of these results has been published previously (Van Essen and Bixby, 1978).

\section{Materials and Methods}

Experiments were made on seven juvenile macaques (Macaca fascicularis, 2 to $3 \mathrm{~kg}$ ). In an initial surgical operation, each animal was sedated with ketamine (10 $\mathrm{mg} / \mathrm{kg}, \mathrm{i} . \mathrm{m}$.) and anesthetized with sodium pentobarbital (20 $\mathrm{mg} / \mathrm{kg}$, i.v.). Under aseptic conditions, the splenium (posterior $1 \mathrm{~cm}$ ) of the corpus callosum was transected, thus interrupting fibers connecting visual cortex in the occipital, temporal, and parietal lobes of opposite hemispheres (Pandya et al., 1971; Hamilton, 1982). In five animals, a Gelfoam pledget $(3 \times 10 \mathrm{~mm})$ soaked in a $25 \%$ solution of horseradish peroxidase (HRP, 20 to $40 \mathrm{mg}$ total; Boehringer Mannheim Biochemical) was placed between the cut surfaces of the callosum. Injections of urea ( 3 to $4 \mathrm{ml} / \mathrm{kg}$ of $25 \%$ urea plus $5 \%$ D-glucose, IV) were made to prevent cortical herniation during surgery. After a survival time of 2 to 6 days and an acute physiological recording session not related to the present study, each animal was perfused through the heart with $4 \%$ paraformaldehyde in $0.1 \mathrm{M}$ phosphate buffer $(\mathrm{pH} 7.4)$ or $1 \%$ formaldehyde, $1.25 \%$ glutaraldehyde, also in phosphate buffer. The brain then was removed, blocked, and equilibrated with buffered $30 \%$ sucrose. Frozen sections were cut at $31 \mu \mathrm{m}$; alternate sets of four sections were stored in phosphate buffer for subsequent HRP histochemistry or in formol/saline for other staining procedures. The diaminobenzidine (DAB) procedure of Graham and Karnovsky (1966) was used for HRP histochemistry. Degenerating terminals were stained by the method of Wiitanen (1969); myelinated fibers were stained by a modified Weigert stain or, in more recent experiments, by the Gallyas (1979) technique, which we have found to yield consistently superior results. In two experiments, injections of $\left[{ }^{3} \mathrm{H}\right]$ proline ( 20 to $30 \mu \mathrm{Ci}$ in $0.1 \mu \mathrm{l}$ of saline) were made into cortex along the $\mathrm{V} 1 / \mathrm{V} 2$ border through a modified hypodermic syringe. Sections were processed for autoradiography following the procedure of Cowan et al. (1972).

Information pertaining to HRP-labeled cells, degenerating terminals, etc., was plotted initially on enlarged photographs of sections. For each staining technique, sections were examined at $0.5-\mathrm{mm}$ intervals ( 1 in 16) unless noted otherwise. The data then were transferred to two-dimensional reconstructions of the cortex made by the procedure described in Van Essen and Maunsell (1980). The density of callosal inputs to various regions was indicated on the maps using the same scale of sparse, moderate, or dense degeneration employed in a previous study (Van Essen and Zeki, 1978). Sections were scored for the degenerating axons and terminals present in layer IV, where the callosal inputs are generally densest and most sharply defined. Occasional regions were encountered in which degenerating terminals were not detectable in layer IV, but degenerating fibers and/or terminals were present in layers $\mathrm{V}$ and $\mathrm{VI}$; in such cases, the degeneration was scored as sparse.

\section{Results}

\section{A standard representation of callosal projections}

Complete reconstructions of the pattern of callosal inputs to extrastriate visual cortex were made for three 


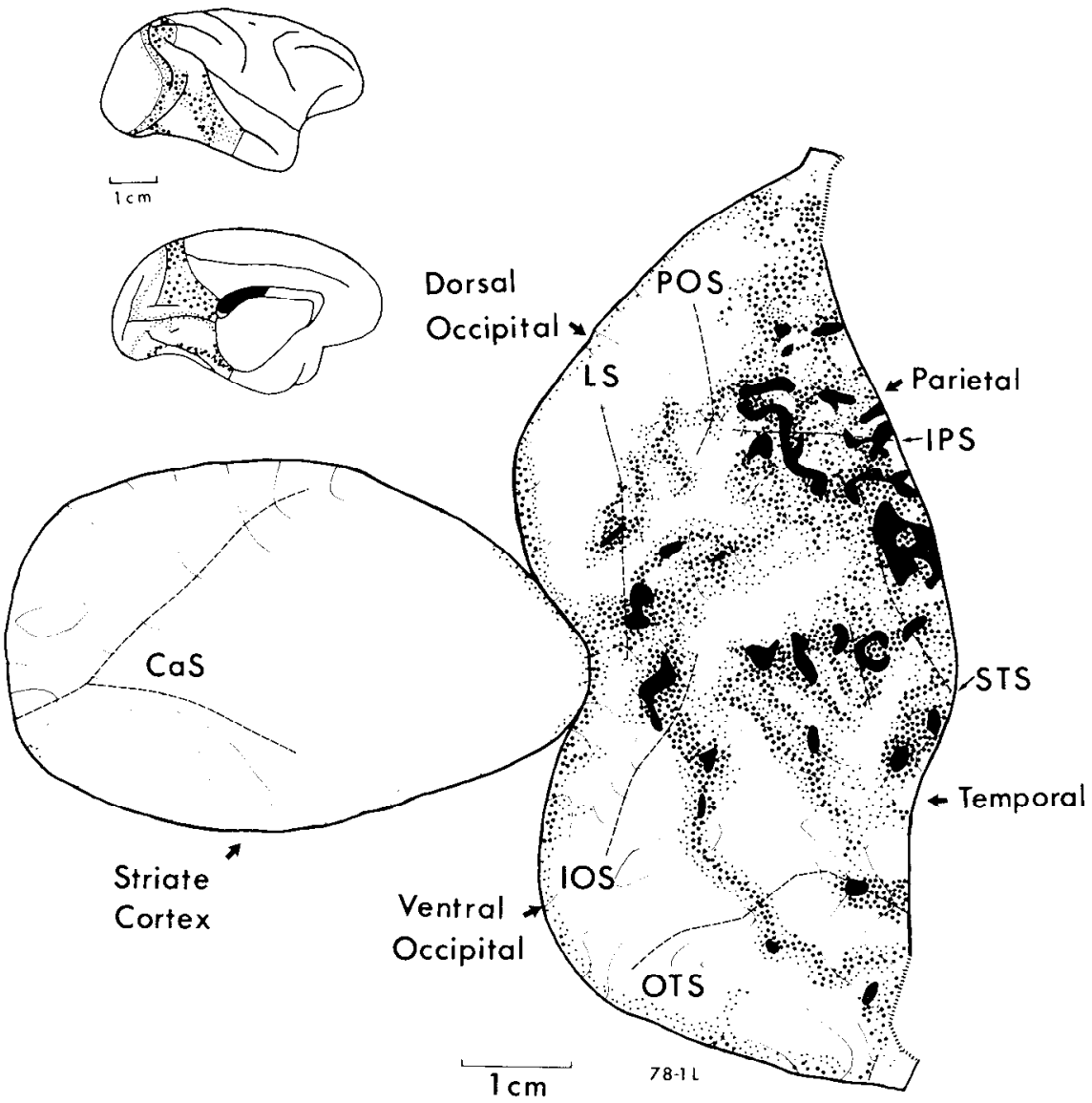

Figure $1 .{ }^{5}$ Callosal inputs to visual cortex in the occipital, parietal, and temporal lobes of the macaque. Sparse, moderate, and dense levels of degeneration resulting from callosal transection are indicated, respectively, by small dots, large dots, and solid black. Each of the fine contour lines within the map represents the outline of layer IV from a single horizontal section, altered in shape so as to permit proper alignment with respect to adjacent contours. The perimeter of the map is formed by artificial discontinuities in the cortical representation (heavy solid lines) and by regions in which the neocortex adjoins allocortical structures (dotted lines). The fundus of each sulcus is denoted by a dashed line within the map. Shown in the upper left are medial and lateral views of the hemisphere; note that, in this and subsequent figures, one of the views is reversed so that dorsal is $u p$ and anterior to the right in both medial and lateral views. The portion of the callosum transected during the initial operation is shown in black. The ventral tip of the splenium was spared inadvertently, but comparison with other hemispheres having complete splenial transection indicates that there are no major gaps in the callosal projections to visual cortex in this hemisphere.

hemispheres, each from a different animal, following splenial transection and the staining of sections for de generating terminals. The basic pattern was similar in all three cases, and we shall begin by describing one of them in detail.

In individual sections, we found the distribution of callosal inputs to be irregular and highly variable at different levels through the cortex, much as has been shown in previous studies (e.g., Myers, 1962; Zeki, 1970). Visualization of the important features of this pattern is most readily achieved by transposing results from a series

"The abbreviations used on the figures are: CaS, calcarine sulcus; IOS, inferior occipital sulcus; IPS, intraparietal sulcus; LS, lunate sulcus; OTS, occipitotemporal sulcus; POS, parieto-occipital sulcus; STS, superior temporal sulcus. of sections onto a two-dimensional cortical map as illustrated in Figure 1. This map contains a representation of the entire occipital lobe plus those portions of the temporal and parietal lobes known to be visual in function. Indicated along the margins of the map are the major regional subdivisions of cortex, including parietal and temporal cortices, dorsal and ventral portions of extrastriate occipital cortex, and striate cortex, which forms the isolated elliptical region on the left of the map. The map was made from the outlines of horizontal sections taken at 2-mm intervals through the cortex; each of the fine contour lines within the map represents one of these sections. Details pertaining to the organization of the map and its relationship to the intact hemisphere are presented in the legends to Figures 1 and 2. A more general description of how such maps are constructed 
and interpreted is provided in Van Essen and Maunsell (1980).

The distribution of degenerating terminals produced by callosal transection is indicated in Figure 1 by small and large dots for sparse and moderate degeneration, respectively, and by solid regions for dense degeneration. It is evident at a glance that there are virtually no callosal inputs to the striate cortex and that the pattern in extrastriatc cortex is highly complex, with large regions essentially devoid of callosal input alternating with zones having an irregular distribution of inputs. An important feature of the overall pattern is that the callosal recipient and callosal free regions of cortex differ in their topological organization. Callosal recipient cortex is arranged as numerous zones of varying widths and lengths, with continuity existing among all zones, whereas the callosal free regions occur as large islands which, in most cases, are separated from one another completely. The callosal recipient zones vary considerably in width (1 to $20 \mathrm{~mm}$ ) and in length $(20$ to $50 \mathrm{~mm}$ ); some are relatively constant in width, but others are highly irregular. 'The dimensions of the major callosal free regions are from 5 to $15 \mathrm{~mm}$ wide and from 10 to $50 \mathrm{~mm}$ long; the cortical surface area that they enclose ranges from 22 to $390 \mathrm{~mm}^{2}$.

Although many aspects of the callosal projection pattern can be analyzed without specific reference to cortical convolutions, it is nonetheless helpful to appreciate how the various callosal recipient zones are related to the location of various gyri and sulci. Some aspects of this relationship can be appreciated just by examining the distribution of callosal inputs to the exposed medial and lateral surfaces of the intact hemisphere (inset, Fig. 1). However, reliance on surface views suffers from the fact that more than half of the extrastriate cortex is buried within sulci. Even when hemisphere drawings are modified to "open up" sulci (Karol and Pandya, 1971), many important details are obscured by inherent inaccuracies in the representation of cortical surface area. More precise comparisons can be made if both callosal inputs and sulcal patterns are displayed on the same cortical map. It is convenient to do this in two steps, the first of which shows only the sulcal pattern and the second of which combines both patterns. Figure $2 A$ shows the representation of sulci on a map of the same hemisphere illustrated in the previous figure, with regions of buried cortex denoted by hatching. As before, the fundus of each sulcus is represented by a dashed line. In Figure $2 B$, the pattern of callosal inputs ( stippling) has been superimposed on the sulcal pattern. For clarity, the contour lines within the map have been deleted, and variations in density of callosal inputs are not shown. In general, one can readily see that callosal recipient cortex occupies about half of the cortex in both gyri and sulci. For the most part, the various callosal recipient strips do not bear any systematic relationship to gyral and sulcal landmarks. The principal exception is the callosal recipient strip adjoining striate cortex, which runs close to the margins of the major sulci in the occipital lobe (the lunate, parietooccipital, inferior occipital, occipitotemporal, and calcarine sulci).

A thorough understanding of Figure 2 requires a detailed comparison of two patterns, each of which is com-



Figure 2. Relationship of callosal projections of gyral and sulcal landmarks. $A$, This shows how cortex within the various sulci ( hatching) is represented on the cortical map. Also shown are the levels at which sections were taken through the hemisphere, with sections $1,4,7$, and 10 specifically noted on both the map and the hemisphere drawings. $B$, The pattern of callosal projections (stippling) has been superimposed on the sulcal pattern to show which portions of callosal recipient cortex lie within sulci and which are exposed to surface view.

plex in its own right and is largely independent of the other; it is hardly surprising that this is not an easy undertaking. Fortunately, though, for many purposes, it is unnecessary to trace the precise relationships between callosal projections and cortical convolutions. For most of the presentation that will follow, the convolutions can largely be ignored, and the cortical maps can be regarded as an abstract, but nonetheless appropriate, format for the analysis of results.

As a prelude to the detailed discussion of callosal projections, it is useful to divide the overall pattern into a set of well defined zones based on the topological relationships among callosal recipient and callosal free regions of cortex. In Figure $3 A$, we have designated nine callosal recipient zones with numerical labels (zones 1 to 9 ) and seven callosal free regions with alphabetical labels (regions $A$ to $G$ ). This provides objective and convenient 
terminology for discussing the relationship between callosal projections and extrastriate visual areas even in regions where the boundaries of visual areas have not been mapped accurately.

Previous anatomical and physiological studies have demonstrated the existence of eight extrastriate visual areas in the macaque. Six of these areas contain topographically organized representations of all or part of the contralateral visual hemifield; these are V2, V3, V3A, V4, the middle temporal area (MT), and the ventral posterior area (VP) (Cragg, 1969; Zeki, 1969, 1971; Van Essen and Zeki, 1978; Ungerleider and Mishkin, 1979; Newsome et al., 1980; Van Essen et al., 1981). Visual areas lacking obvious topographic organization include inferotemporal cortex (IT) (Gross et al., 1972; Desimone and Gross, 1979) and posterior parietal cortex (area 7) (Lynch et al., 1977; Robinson et al., 1978). Five of the topographically organized areas (V2, V3, V3A, V4, and VP) have borders which are known to be closely related to major callosal recipient zones as indicated in Figure $3 B$ (Zeki, 1971, 1978a, b; Van Essen and Zeki, 1978; Newsome et al., 1980). Precise determination of their borders in any given hemisphere generally has proven very difficult, however. MT is the only extrastriate area whose borders have as yet been determined accuratcly and completely on the basis of architectonic criteria (Van Essen et al., 1981), and its location is indicated in Figure $3 B$ by a solid outline. For the other areas, the approximate boundaries are indicated by dashed lines in the figure and are discussed in greater detail below.

Regions $A$ and B: Visual areas V2, V3, and VP. Regions $\mathrm{A}$ and $\mathrm{B}$, the two largest callosal free regions in extrastriate cortex, occupy a broad belt which almost completely surrounds the striate cortex and is separated from it by narrow callosal recipient strips (zone 1 dorsally and zone 2 ventrally). Previous studies have shown that striate cortex projects to two topographically organized visual areas, V2 and V3, within regions $\mathrm{A}$ and $\mathrm{B}$ (Zeki, 1969, 1970; Cragg, 1969). Along its posterior border, V2 adjoins striate cortex, and the two areas share a common vertical meridian representation that coincides with callosal recipient zones 1 and 2 . V2 contains distinct dorsal and ventral subdivisions joined only at the region of foveal representation. Dorsal V2 occupies most of region A, contains a representation of inferior visual fields, and adjoins the region of inferior field representation in striate cortex. Ventral V2 occupies most of region B, contains a representation of superior visual fields, and adjoins the region of superior field representation in striate cortex. The horizontal meridian is represented along the anterior margin of V2 in both dorsal and ventral subdivisions; these borders are situated slightly posterior to callosal recipient zones 3 and 4 . Originally, it was thought that V3 adjoined V2 along its entire length, sharing a common horizontal meridian representation with it (Zeki, 1969; Cragg, 1969). However, more recent studies have suggested a different arrangement in which V3 is restricted to the dorsal half of the hemisphere, with a separate area adjoining the ventral subdivision of V2. A key argument against the original scheme is the finding of a marked asymmetry in the projections of striate cortex to regions A and B. Dorsal striate cortex projects to two distinct areas, V2 and V3, within region $\mathrm{A}$, whereas ventral striate cortex projects only to V2 in region $B$ and not to the region immediately anterior to it (Van Essen et al., 1979; D. C. Van Essen and W. T. Newsome, manuscript in preparation). In addition, the myeloarchitecture of V3 differs from that of cortex adjoining ventral V2 (Newsome et al., 1980; D. C. Van Essen and W. T. Newsome, manuscript in preparation). Coupled with this
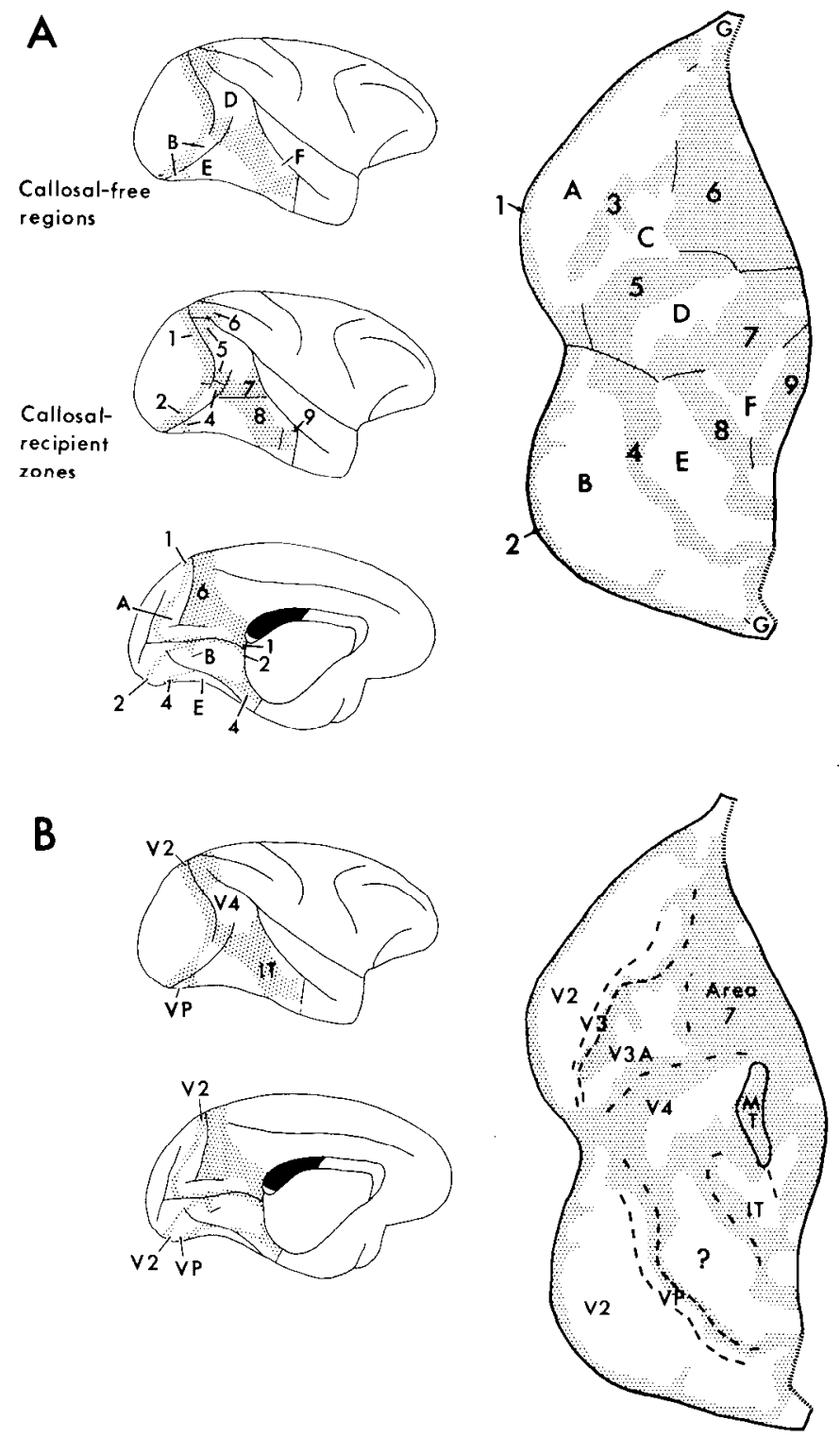

Figure 3. Relationship of extrastriate visual areas to callosal recipient zones and callosal free regions. $A$, Major callosal recipient zones ( 1 to 9 ) and callosal free regions ( $A$ to $G$ ) are designated on the cortical map and on the hemisphere drawings. The borders between callosal recipient zones (fine lines) are somewhat arbitrary but, when possible, have been drawn in a manner most likely to reflect the boundaries between visual areas. $B$, This shows the location of known visual areas on the cortical map (right) and in the intact hemisphere (left). Boundaries identified by architectonic criteria are indicated by solid lines (i.e., MT and V1/V2 borders). Other borders are denoted by dashed lines; the spacing between dashes reflects the accuracy with which the various border assignments can be made on the basis of available evidence. 
is a pronounced asymmetry in the organization of callosal recipient zones 3 and 4 (Fig. $3 B$ ). Zone 3 , which forms the anterior border of $\mathrm{V} 3$, terminates abruptly in the lateral part of the lunate sulcus next to the juncture of regions $\mathrm{A}$ and $\mathrm{C}$. In contrast, zone 4 is continuous with other callosal recipient zones both laterally, where it joins zone 5 , and medially, where it joins zone 2 . 'These results suggest that the region adjoining V2 ventrally should be considered distinct from V3. Further evidence supporting this suggestion comes from the striking similarities in topographic organization and callosal inputs between the region adjoining ventral V2 in the macaque (Newsome et al., 1980; D. C. Van Essen and W. T. Newsome, manuscript in preparation) and the comparable region in the owl monkey, where a distinct visual area, the ventral posterior area (VP) has been identified (Newsome and Allman, 1980). On the basis of the apparent homology indicated by these similarities, we have adopted the same name, VP, for this area in the macaque.

Previous studies combining electrophysiological mapping of receptive field locations with anatomical demonstration of callosal projections have shown that the vertical meridian is represented along the anterior border of V3 (i.e., on the side away from V2) and that this border is located along callosal recipient strip 3 (Zeki and Sandeman, 1976; Van Essen and Zeki, 1978). However, significant uncertainties about the borders of V3 remain. It has not been established exactly how far V3 extends laterally (within the lunate sulcus) and medially (within the parieto-occipital sulcus). In addition, there is significant uncertainty concerning the exact location within zone 3 of the border between V3 and V3A. Zeki (1978b) has suggested that, within zone 3 , a reduced density of callosal inputs often occurs right at the V3/V3A border. However, no independent evidence is provided that this is indeed where the border is situated; moreover, we have not found a central "channel" of reduced callosal input within zone 3 to be a common feature (see Figs. 1 and 5 and also Figs. 2 to 4 of Van Essen and Zeki, 1978). Similar difficulties arise in trying to determine the border of VP in region $\mathrm{B}$, although the uncertainties are smaller because callosal recipient zone 4 is narrower and more uniform over most of its length.

Two additional features pertaining to the callosal recipient zones surrounding regions $\mathrm{A}$ and $\mathrm{B}$ are worth mentioning. First, zones 1 and 2 together surround most of striate cortex, but they do not envelop it completely. The gap along the upper and lower margins of the map (region G) is small, but it is significant because of its relationship to the representation of the visual periphery and will be discussed in more detail below. Second, zones 1 and 2 merge in three places with other callosal recipient zones, namely, with zone 6 at the top of the map, zone 5 in the center, and zone 4 at the bottom. The juncture with zone 5 occurs close to the foveal representation in striate cortex and V2. A similar confluence of callosal recipient zones near the region where the center of gaze is represented has been reported in the cat, bushbaby, rat, and mouse but is not found in the owl monkey (Ebner and Myers, 1965; Newsome and Allman, 1980; Cusick and Lund, 1981).

Region C: Area V3A. Region C of callosal free cortex is small, irregularly shaped, and completely buried within the lunate and parieto-occipital sulci. Physiological recordings from this region have shown that it contains a topographically organized area, V3A, which is distinct from both V3 on its medial side and V4 on its lateral side (Van Essen and Zeki, 1978). Within V3A, inferior fields are represented medially and superior fields are represented mainly laterally, but there are significant complexities in its topographic organization. It is uncertain whether additional visual areas are present within region $\mathrm{C}$, as its anterior portion (in the parieto-occipital and intraparietal sulci) has not been studied in detail.

Region D: V4 and $M T$. Callosal free region D lies mainly on the pre-lunate gyrus and the posterior bank of the superior temporal sulcus, with a small extension into the dorsal tip of the inferior occipital sulcus. Surrounding it are callosal recipient zones 4 to 8 in the inferior occipital sulcus, lunate sulcus, pre-lunate gyrus, superior temporal sulcus, and inferior temporal gyrus. Two topographically organized visual areas, V4 and MT, have been identified within region $\mathrm{D}$ and the adjacent callosal recipient cortex. MT lies almost entirely within zone 7 , with only a small portion extending into region D (Van Essen et al., 1981). MT contains a complete representation of the contralateral visual hemifield but with substantial disorderliness in the representation (Zeki, 1978d; Montero, 1980; Van Essen et al., 1981). The callosal inputs to MT are widespread and irregular (cf., Fig. 14, Van Essen et al., 1981), but whether this is related to the disorderliness in topographic organization remains to be determined.

V4, identified on the basis of the major projection that it receives from $\mathrm{V} 2$, is known to occupy much of zone 5 and region D (Zeki, 1971, 1978a), but its full extent has proven difficult to determine. One simple hypothesis is that V4 occupies all of region D plus a surrounding strip of callosal recipient cortex. The available anatomical and physiological evidence is consistent with this hypothesis but also with alternative possibilities in which V4 is either much larger or much smaller. The key observations are (i) that most or all of region $\mathrm{D}$ and the immediately adjoining callosal recipient cortex receives inputs from dorsal V2 (Zeki, 1971; A. Burkhalter, W. T. Newsome, and D. C. Van Essen, unpublished evidence); (ii) that there is little or no input to this region from ventral V2 (A. Burkhalter, W. T. Newsome, and D. C. Van Essen, unpublished evidence); (iii) that there is little or no representation of superior visual fields in this region (Zeki, 1977; Van Essen and Zeki, 1978; Van Essen et al., 1981); and (iv) that there are inhomogeneities in this region with respect to its architecture, topographic organization, and functional properties (Zeki, 1971, 1977, 1978c; Van Essen and Zeki, 1978; Schein et al., 1982). Thus, one could plausibly argue (i) that V4 occupies all of this region but is internally complex and lacks a complete representation of the visual field; (ii) that V4 occupies only part of this region, with an additional area interposed between it and MT; or (iii) that V4 occupies all of this area and more, extending into region $\mathrm{E}$ ventrally (see below). Until such issues are resolved, it will be difficult to evaluate questions pertaining to possible homologues of V4 in other species, such as the dorsolateral area (DL) in the owl monkey (Allman and Kaas, 
1974), since such suggestions are based, in part, on proximity to other visual areas (MT and V2).

Regions $E$ and $F$ : Callosal recipient zones 6 to 9. Region E, which extends across portions of the inferior temporal gyrus, occipitotemporal sulcus, and occipitotemporal gyrus, has not been studied in detail even though it is the third largest callosal free region in extrastriate cortex. In preliminary studies, we have found that this region is visually responsive and topographically organized and that it receives direct inputs from ventral V2 (D. C. Van Essen, W. T. Newsome, and A. Burkhalter, unpublished evidence; see also Desimone and Gross, 1979). Desimone et al. (1980) have found projections to inferotemporal cortex originating from at least part of this region as well as a large part of what is clearly V4 in the dorsal part of the hemisphere. This is consistent with the possibility mentioned above that V4 extends into region $\mathrm{E}$, but it certainly does not rule out the possibility that region $\mathrm{E}$ contains one or more heretofore unidentified areas. In any event, region $\mathrm{E}$ plus the six areas already discussed, constitute all of the known topographically organized extrastriate visual cortex in the macaque. In addition, there are several important regions of visually responsive cortex in which there is little or no discernible topographic organization. These include inferotemporal cortex, posterior parietal cortex, and a visually responsive region medial to MT.

Inferotemporal cortex (I'T) is located in the vicinity of region $\mathrm{F}$ and parts of the surrounding callosal recipient zones 7, 8, and 9 (Gross et al., 1972; Desimone and Gross, 1979). Within IT, however, there are regional differences in architecture (Seltzer and Pandya, 1978), connections (Zeki, 1973; Seltzer and Pandya, 1978; Desimone et al., 1980 ), and receptive field size (Desimone and Gross, 1979), suggesting that there may be more than one functionally distinct subdivision of this region. Posterior parietal cortex (area 7, or PG and PE) occupies much of callosal recipient zone 6 (Lynch et al., 1977; Robinson et al., 1978). As with inferotemporal cortex, there are significant regional variations in architecture and connections of posterior parietal cortex (Seltzer and Pandya, 1980); hence, this region is also likely to contain more than one functional subdivision. The third visual region lacking obvious topographic organization is a strip of cortex adjoining MT near the fundus of the superior temporal sulcus (Van Essen et al., 1981). This region differs from MT in its myeloarchitecture, its lack of inputs from striate cortex, and in the large size of its receptive fields, and it differs from neighboring areas $\mathrm{V} 4$ and IT because it has a high incidence of direction selective cells. The possibility exists, however, that it is functionally a part of posterior parietal cortex, or a subdivision thereof, since most visually responsive neurons in the inferior parietal lobule (i.e., lateral area 7) are also direction selective and have large receptive fields (Motter and Mountcastle, 1981). Anterior to the parietal and temporal visual areas is a belt of polysensory cortex which separates visual cortex from auditory and somatosensory areas (Desimone and Gross, 1979; see Fig. 11 in Van Essen and Maunsell, 1980).

Region G: The prostriate area. Region $\mathrm{G}$ is the smallest of the callosal free regions shown in Figure 3, and it is also the only one which directly adjoins striate cortex without an intervening strip of callosal recipient cortex. It is located at the anterior tip of the calcarine sulcus next to the hippocampal formation. The relationship of region $\mathrm{G}$ to striate cortex can be demonstrated most clearly when results are displayed on a modified cortical map in which the continuity along the striate/extrastriate border in the calcarine sulcus is preserved (Fig. 4). The transformation from our standard cortical map to this modified representation is illustrated in Figure $4 A$. It involves the transposition of two regions at the upper and lower extremes of the standard map to a location on the far left of striate cortex, thereby eliminating the artificial discontinuities in this part of the representation. The distribution of callosal inputs to calcarine cortex is shown in Figure $4 B$. Callosal free region $G$ is relatively small ( 6 to $7 \mathrm{~mm}$ long and 2 to $3 \mathrm{~mm}$ wide), and it is located just alongside the representation of the temporal periphery in striate cortex (Daniel and Whitteridge, 1961). This makes intuitive sense, inasmuch as one would not expect callosal inputs immediately next to a region in which receptive fields are all far from the vertical meridian. Also, a similar gap in commissural inputs to the border of medial striate cortex has been found in several other species (Ebner and Myers, 1965; Benevento and Ebner, 1971; Newsome and Allman, 1980; Cusick and Lund, 1981). It is interesting that the cortex in region $G$ has a distinctive architecture, characterized by its sparse myelination and poorly developed layer IV. The stippled portion in Figure $4 B$ corresponds to the prostriate area previously identified by Sanides (1970). A final point worth noting about this region is that it occurs right at the narrowest part of extrastriate cortex, forming a thin strip separating striate cortex on one side and allocortex (presubicular and retrosplenial areas) on the other.

\section{Individual variability in callosal projections}

In view of the usefulness of the callosal projection pattern as a basis for determining the location of numerous extrastriate visual areas, it is obviously important to know how much individual variability there is in this pattern. In a previous study, a significant degree of variability was found in the callosal projections to the dorsal half of the occipital lobe (Van Essen and Zeki, 1978). To provide a more extensive basis for comparison, we mapped the callosal projections to all of extrastriate visual cortex in two additional hemispheres as illustrated in Figure 5. Both maps were made from sections cut in the parasagittal plane, and the orientation of contour lines is therefore quite different from the example shown previously, in which the hemisphere was cut in the horizontal plane. In all three maps, however, the relative locations of gyri and sulci are very similar.

From comparison of the maps in Figures 1 and 5, it is evident, on the one hand, that there are many basic features common to all three callosal projection patterns. On the other hand, there are substantial individual differences in detailed organization. The similarities and differences can be discussed more readily with reference to Figure 6, which shows maps of callosal recipient cortex for all three hemispheres examined in this study along with labels for the major callosal recipient and callosal 
A
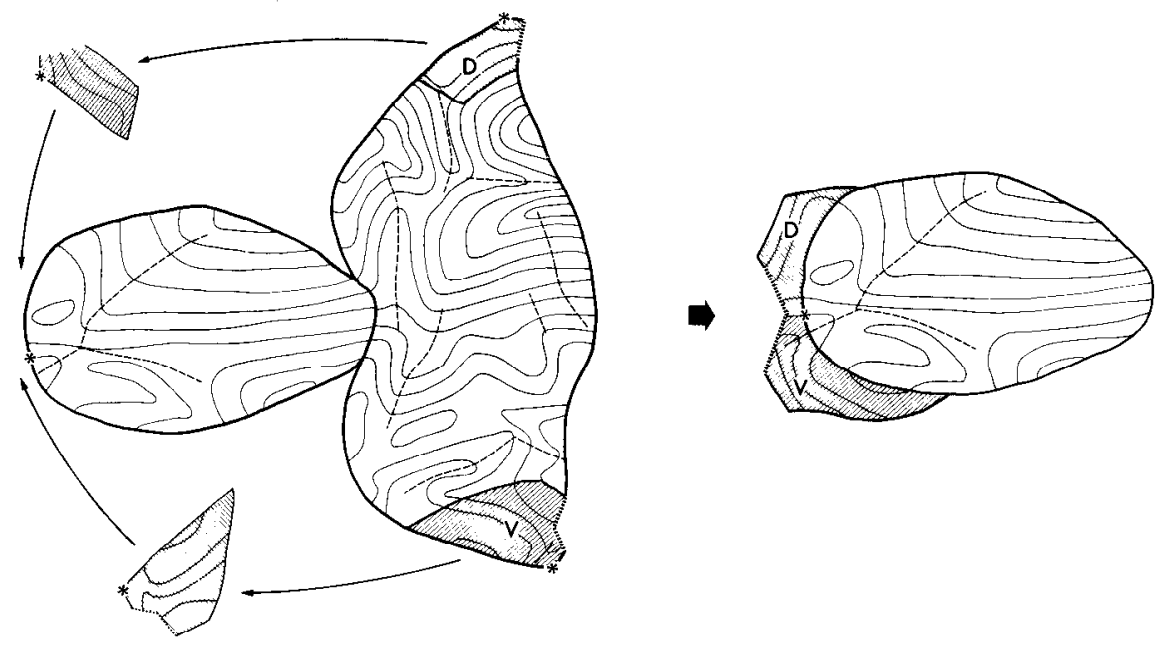

B

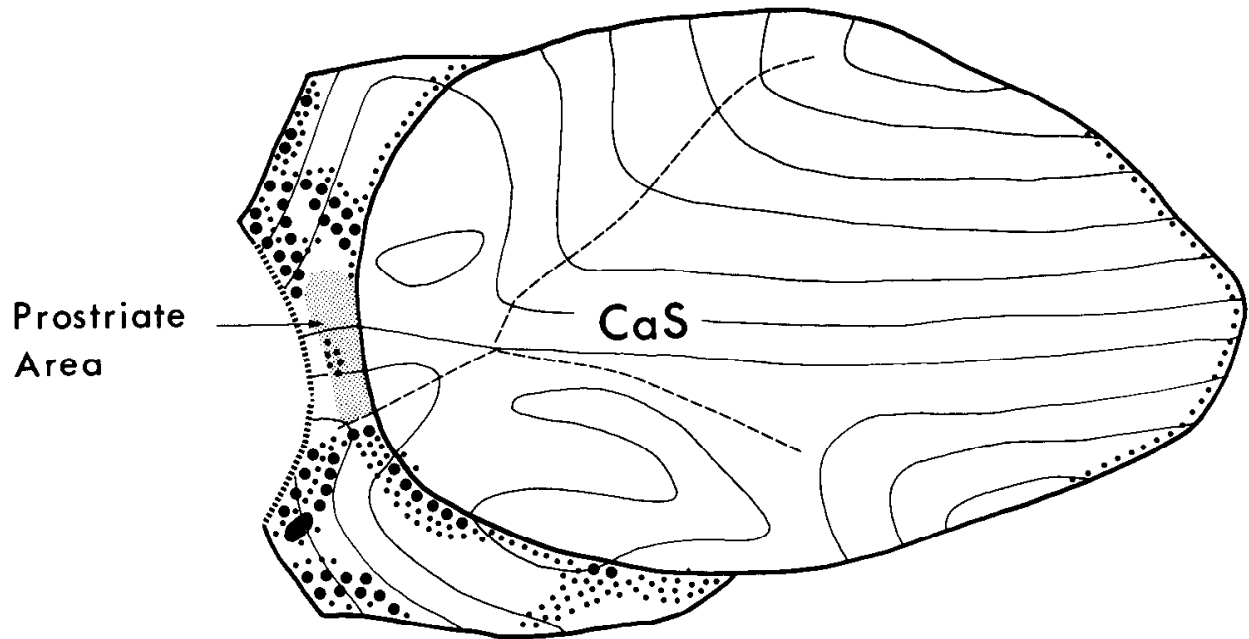

$1 \mathrm{~cm}$

Figure 4. Callosal inputs to the calcarine sulcus. $A$, The representation of extrastriate cortex in and near the anterior limb of the calcarine sulcus (hatched regions) can be transposed in a manner that restores the continuity of dorsal and ventral subdivisions ( $D$ and $V$ ) with each other and with striate cortex. $B$, With the aid of this modified representation, it is easier to see the organization of callosal recipient and callosal free regions in the anterior calcarine sulcus. The architectonically distinct prostriate area (stippling) adjoins striate cortex along the representation of the far periphery and separates dorsal and ventral subdivisions of V2. Retrosplenial and presubicular cortex occupy the narrow strip between the prostriate area and the hippocampal formation (dotted boundary on left).

free regions. In all three maps, it is easy to recognize callosal free regions $\mathrm{A}, \mathrm{B}, \mathrm{D}, \mathrm{E}$, and $\mathrm{G}$ and callosal recipient zones $1,2,4$, and 6 as originally delineated in Figure 3 . The strongest similarities are in ventral occipi- tal cortex, where callosal recipient zones 2 and 4 and the adjoining callosal free regions $\mathbf{B}$ and $\mathbf{E}$ are organized in very similar fashion in all three maps. Identification of other callosal recipient and callosal free regions is more 


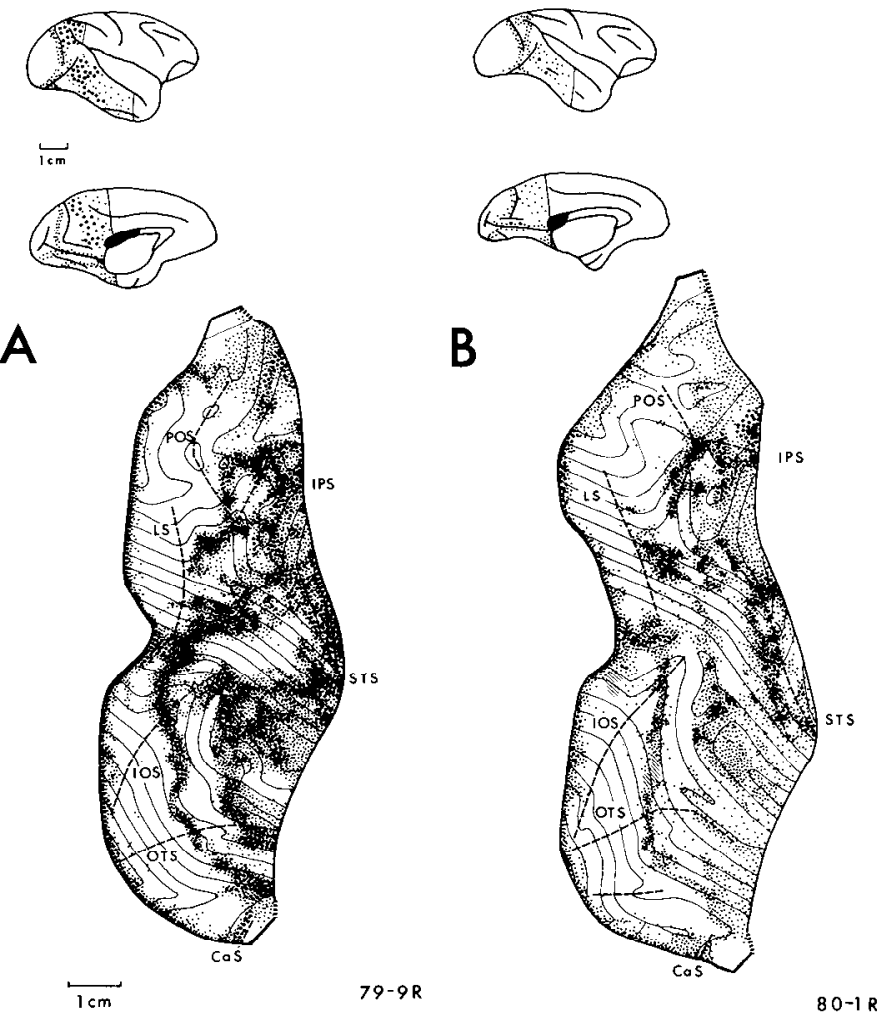

Figure 5. Individual variability in callosal projections. $A$ and $B$ show callosal inputs to two hemispheres from different animals. Both hemispheres were sectioned in parasagittal plane so that the contour lines on the maps differ greatly from those in the map of Figure 1 but are similar to those illustrated previously for a parasagittal map (Fig. 5 in Van Essen and Maunsell, 1980). The hatched regions in the vicinity of the IOS and OTS in the right map indicate sites at which degeneration could not be scored due to local cortical damage from disruption of a blood vessel during the acute physiological recording session made in this hemisphere. In monkey $80-1 \mathrm{R}$, the brain was fixed with a glutaraldehyde/formaldehyde mixture rather than the straight formaldehyde fixation used for the other two hemispheres shown in this figure and Figure 1. This probably accounts for the lower overall density of degeneration found in this hemisphere, but it is unlikely to account for the significant differences in the pattern of projections.

difficult because of differences in the topological organization of the callosal projection pattern and differences in the relationship of this pattern to gyral and sulcal landmarks.

It is worthwhile to comment on the more prominent of these differences, because they bear importantly on the issues of variability in the location of visual areas and of the confidence with which areal boundaries can be identified. The most striking differences are in the upper halves of the maps, corresponding to dorsal extrastriate cortex. For example, in maps $I I$ and $I I I$ of Figure 6, there is no callosal recipient zone strictly comparable to zone 3 of map I (i.e., one which is highly elongated and connected to other zones only at its medial (upper) end). Nevertheless, it is possible to recognize callosal recipient zones in maps $I I$ and $I I I$ which presumably correspond to zone 3 by virtue of their location and orientation and which therefore denote the anterior border of V3. They differ mainly in being continuous with zones 5 and 6 at multiple points. As a result of these topological differences, the cortex which presumably corresponds to callosal free region $\mathrm{C}$ (area V3A) is organized as distinct medial and lateral subregions, $C_{m}$ and $C_{l}$, in maps $I I$ and III. It is interesting that, of the three examples studied previously (Van Essen and Zeki, 1978), one hemisphere had a pattern similar to that in map I (i.e., with a wide strip of callosal free cortex interconnecting regions $A$ and C), whereas the other two had patterns more like those in map II. Thus, the pattern in map II may be the most common, but the variability remains substantial even when a larger sample is examined.

Similar considerations apply to the variability in callosal recipient zone 5 and the adjoining callosal free region D. Maps $I$ and $I I$ in Figure 6 are very similar in this region, whereas map III has a markedly different pattern in which there is a large callosal recipient patch that cannot be unambiguously assigned to either zone 3 or zone 5 and a large callosal free region that might belong either to region $\mathrm{A}$ or to region $\mathrm{D}$. Although this leads to an unusually large degree of uncertainty in assigning borders to several areas (including V3, V3A, and V4) in this hemisphere, it should be emphasized that the uncertainties would be even greater in the absence of any information about callosal projections. The pattern in this part of map III appears to be a genuinely aberrant one anyway, as all three hemispheres examined previously (Van Essen and Zeki, 1978) conformed to the pattern of maps $I$ and $I I$, which we therefore take to be the standard.

A third region showing pronounced individual variability is in the vicinity of callosal recipient zones 7 and 8 in the temporal lobe. In maps $I I$ and $I I I$, there is no callosal free region that clearly corresponds to region $\mathrm{F}$ in map $I$. Hence, callosal recipient zone 9, identified in map $I$ as the zone immediately anterior to region $\mathrm{F}$, cannot be designated using the same criterion in maps II and III. Analysis of this region is complicated further by the variability in location of callosal free region $D$. In maps $I$ and $I I$, region $\mathrm{D}$ is highly elongated, running from the fundus of the inferior occipital sulcus almost to the fundus of the superior temporal sulcus; its long axis is oriented roughly horizontally in the hemisphere and left to right on the map. In map III, region D is similar in size, equally elongated, and centered about a similar location on the cortical map, but its orientation is closer to vertical both in the map and in the intact hemisphere. Although it is still possible to recognize zones corresponding to callosal recipient strips 7 and 8 in map $I I I$, it is obviously more difficult to establish objective criteria for determining how far each zone extends.

The aforementioned examples are by no means exhaustive, but they suffice to illustrate several general points about individual variability in callosal projection patterns. Clearly, the variability is large enough that, for many purposes, it is preferable to know the callosal projection pattern for the hemisphere under examination, rather than relying on comparison with a standardized representation. Some of the pattern differences are topological in nature; others are geographical; i.e., they concern the distribution of inputs with respect to gyral and sulcal landmarks. We emphasize that these differences are not artifactual by-products of the technique of 

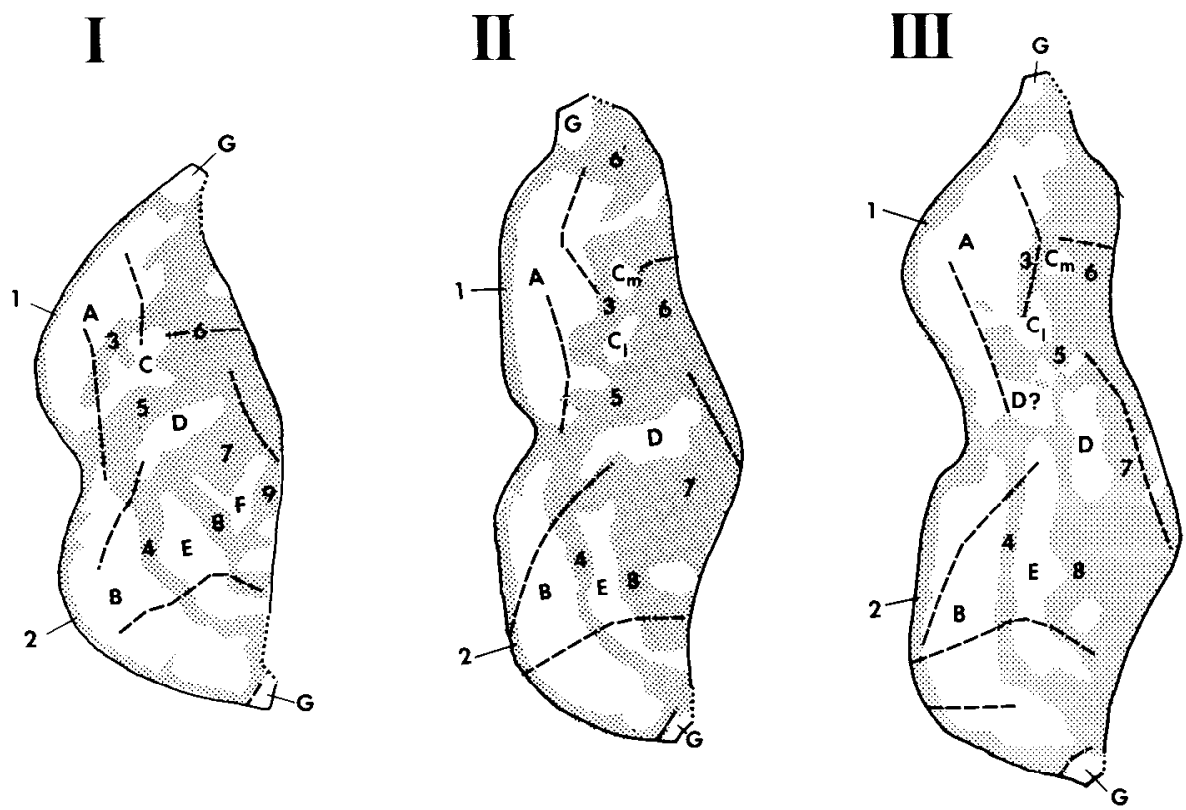

Figure 6. Comparison of callosal recipient zones and callosal free regions in the three hemispheres illustrated in Figures 1 and 5. The three patterns are broadly similar but show marked individual variability in details of their topological organization and in their relationship to gyral and sulcal landmarks. Regions in which pronounced individual differences were present were carefully re-examined (with staining of additional sections when necessary) to insure that the variability was not due to staining artifacts.

constructing cortical maps; rather, they reflect genuine differences that can also be seen by comparing results displayed on sections or on drawings of the intact hemisphere. It is difficult to quantify the overall degree of variability, but an approximate rule of thumb is that the location of any particular feature of the callosal projection pattern with respect to other features of the pattern or to geographical landmarks can vary by anywhere from a few millimeters to as much as a centimeter in different individuals.

\section{Relationship of callosal inputs to visual topography}

The preceding sections have dealt mainly with the overall pattern of callosal inputs and the relationship of this pattern to the various functional subdivisions that make up extrastriate cortex. In the present section, we examine the complexities of callosal projections that are evident at a finer grained level. When examined closely, all of the callosal recipient zones show at least some degree of local irregularity in their internal organization, and in many instances, the spatial fluctuations in the density of callosal inputs are both large and abrupt. Moreover, these variations in "fine structure" are highly dissimilar from one individual to the next. They are greatest in regions well away from the striate cortex but are present even in the callosal recipient zone adjoining it.

An obvious question is whether the local variations in density of callosal inputs are related to local irregularities in the representation of the vertical meridian. In a previous study involving electrophysiological recordings combined with anatomical mapping of callosal inputs, a weak correlation between the density of callosal inputs and the distance of receptive fields from the vertical meridian was demonstrated within callosal recipient zone 5 (areas V3A and V4; Van Essen and Zeki, 1978). In order to obtain further evidence bearing on this issue, we have compared the irregularities in two overlapping sets of cortical connections: the callosal pathway and the ipsilateral projections from regions of vertical meridian representation. In two experiments, a single injection of $\left[{ }^{3} \mathrm{H}\right]$ proline was made at the ventral border between $\mathrm{V} 1$ and V2, where the superior vertical meridian is represented (Daniel and Whitteridge, 1961). In these experiments, the splenium of the corpus callosum also was transected, and neighboring sections were, respectively, stained for degeneration and processed for autoradiography at 0.25 $\mathrm{mm}$ intervals. The $\left[{ }^{3} \mathrm{H}\right]$ proline injections led to anterogradely transported label in several cortical areas in the ventral part of the hemisphere, but in the present context, the particular projections of interest are those within V2 (Fig. 7A) and those along the anterior border of VP (Fig. $7 B$ ). In both cases, the transported label was distributed in a patchy fashion that was closely related to local irregularities in the pattern of callosal inputs. In Figure $7 \mathrm{~A}$, the injection at the V1/V2 border (solid black) resulted in anterograde transport (hatching) to cortex 1 to $2 \mathrm{~mm}$ around the injection site plus a separate patch (arrow) about $3 \mathrm{~mm}$ from the center of the injection and still clearly within V2. Interestingly, this patch coincides precisely with a protrusion of callosal recipient cortex (stippling) that extends several millimeters from the V1/V2 border. Such protrusions are a common feature of callosal projections to the V1/V2 border, as they are present in all three hemispheres illustrated in Figures 1 and 5 as well as in the three hemispheres examined previously (Van Essen and Zeki, 1978). Their location along the V1/V2 border is highly variable, though, and 

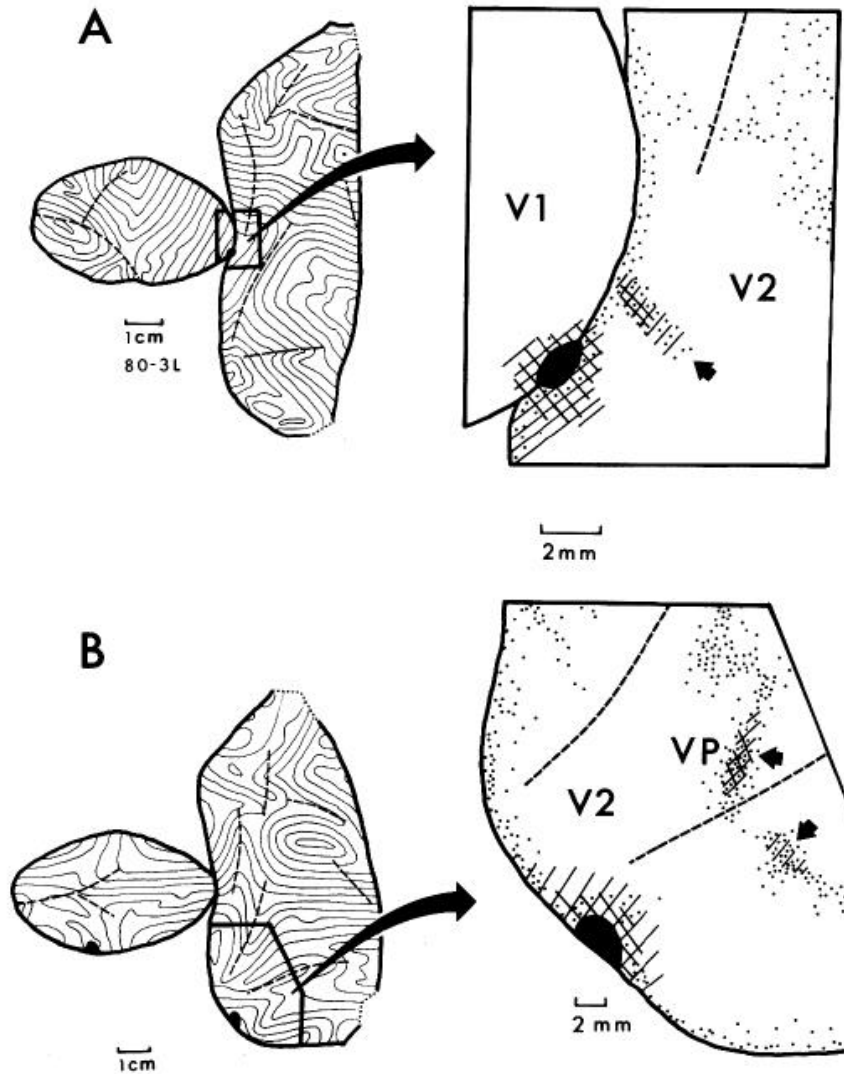

$$
2 \mathrm{~mm}
$$

$$
79.91
$$

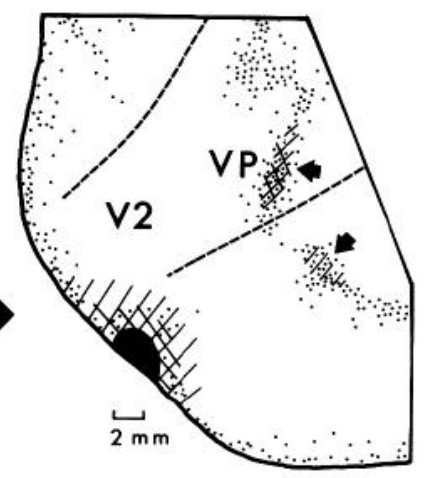

Figure 7. Coincidence of irregularities in interhemispheric and intrahemispheric connections. $A, \mathrm{~A}\left[{ }^{3} \mathrm{H}\right]$ proline injection (black region) at the V1/V2 border near the region of foveal representation resulted in anterograde transport (hatching) to a distinct patch (arrow) several millimeters from the injection site but still within V2. This site coincided with a distinct protrusion of callosal recipient cortex from the V1/V2 border. We considered the possibility that the coincidence of degeneration and transported label was an artifact resulting from damage produced at the site of the tracer injection. This is unlikely, however, because very little damage was produced by this injection and also because, in other experiments, no degeneration was seen at targets associated with injections well away from the vertical meridian representation (except in occasional instances in which the injection produced very obvious damage). $B$, In a separate experiment, another injection along the ventral V1/V2 border revealed patchy projections to VP which coincided with irregularities in the density of callosal inputs to this region.

we have not discerned any systematic relationships with respect to superior versus inferior or central versus peripheral representations.

In the experiment illustrated in Figure $7 B$, there were no obvious irregularities in the labeling pattern within V2, but the transport to VP occurred as two distinct patches having a center-to-center spacing of more than $6 \mathrm{~mm}$ (arrows). Once again, the patchy anterograde transport is correlated with irregularities in callosal projections, as the cortex between the patches receives much sparser callosal inputs than the cortex within the patches. Obviously, these data are not sufficient to reveal exactly how the visual field is represented in the vicinity of the callosal projection irregularities. The point that we wish to emphasize, however, is that the close correlation between inter- and intrahemispheric connections suggests that the fine structure of each pattern reflects important aspects of cortical organization (see also "Discussion").

\section{Cells of origin of the callosal projection}

In order to obtain retrograde labeling of callosal projection neurons, HRP was applied to the cut surface of the callosum, as described under "Materials and Methods." Successful labeling of cells over extensive portions of extrastriate cortex was obtained in five hemispheres from three animals. The great majority of labeled neurons were pyramidal cells, most commonly in layer III but also in layers II, V, VI, and IV, in order of decreasing occurrence. Figure 8 shows a dark-field photomicrograph of one such cell. As shown by Jacobson and Trojanowski (1974) for callosal projection cells in the frontal lobe, we found retrograde labeling of small and medium size pyramidal cells along with large pyramids. Thus, callosal projection cells in the macaque cannot be identified on the basis of cell size alone, although they may, nonetheless, be larger on average than cells which do not project interhemispherically.

In most experiments, we tried to optimize retrograde labeling by keeping the survival time after callosal transection to 2 or 3 days, which is too brief to allow staining of degenerating terminals. In one experiment, however, we obtained successful retrograde labeling with a survival time of 6 days, which permitted direct comparison of the distribution of the terminals and cells of origin of the callosal pathway. Figure 9 shows drawings of adjacent horizontal sections taken from this hemisphere and stained for degeneration (left) or reacted for HRP

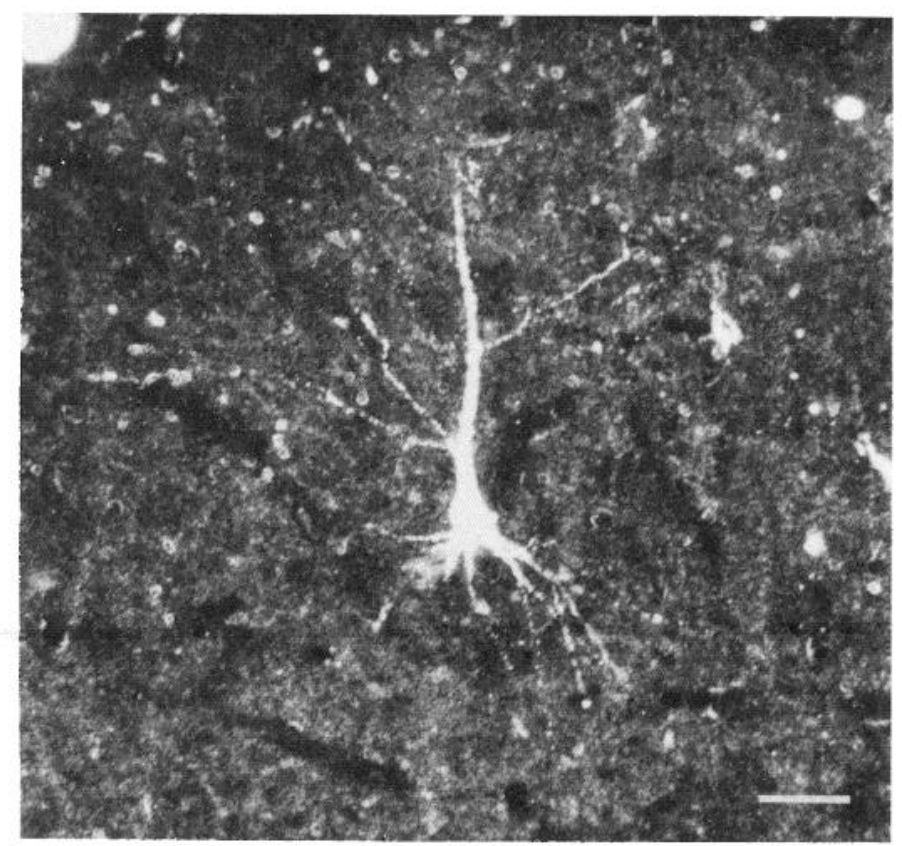

Figure 8. A retrogradely labeled callosal projection cell in cortical layer III identified after HRP application to the cut surface of the callosum. Several basal dendrites as well as a stout apical dendrite can be seen emanating from the $12-\mu \mathrm{m}$ soma of this pyramidal cell. Dark-field illumination was used. Scale bar, $20 \mu \mathrm{m}$. 
(right). Variations in the density of degeneration are denoted by the degree of stippling in the drawing on the left. On the right, small dots represent individual retrogradely labeled cells in layer II or III, large dots represent clusters of five such superficially located cells, and crosses represent labeled cells in layer V or VI. Although the two patterns are not identical, they are similar in several important respects. In this and all other pairs of sections that we examined, the great majority of callosal projection cells lay in callosal recipient cortex as determined from inspection of adjacent degeneration-stained sections. Only in rare instances were callosal projection cells found more than $2 \mathrm{~mm}$ from callosal recipient cortex. In many regions, there was a clear correspondence in the local variations in density of callosal inputs and outputs. For example, in Figure 9, there are three patches of labeled cells in the medial part of the superior temporal sulcus which coincide with three patches of moderate-toheavy degeneration (arrows). The major difference in the patterns is the absence of labeled cells in two of the callosal recipient patches, one at the lateral V1/V2 border and the other in the medial part of the lunate sulcus (asterisks). These gaps almost certainly reflect nonuniform uptake of HRP by cut axons in the callosum, since in other experiments, both regions showed substantial retrograde labeling despite incomplete labeling in a different set of known callosal recipient zones.
The experiment in which we obtained the most extensive retrograde labeling in extrastriate cortex is illustrated in Figure 10. It is unlikely that we labeled more than a small fraction of the total population of callosal projection cells in any given region. Nevertheless, the overall distribution of labeled cells is broadly similar to the pattern of callosal fiber terminations found in other hemispheres (inset). In particular, it is possible to identify zones of callosal projection neurons corresponding to callosal recipient zones 1,3 , and 6 in the dorsal half of the hemisphere and zones 2,4 , and 7 in the ventral half. Some of these zones, most notably 1,2 , and 5, are not labeled in their entirety in this hemisphere, but the gaps presumably reflect nonuniform HRP uptake as argued above.

The one consistent disparity that we noted in the extent of callosal inputs and outputs was in inferotemporal cortex. This region, which receives major callosal inputs (zones 8 and 9), had no more than a few labeled cells in any of the hemispheres that we examined. This result is not surprising, though, because previous anatomical studies (Jouandet and Gazzaniga, 1979; Hamilton, 1982) have shown that the interhemispheric projections of inferotemporal cortex pass through the anterior commissure and not the corpus callosum. Comparison of our results with those of Jouandet and Gazzaniga (1979) suggests that there is considerable overlap in the fields
DEGENERATION

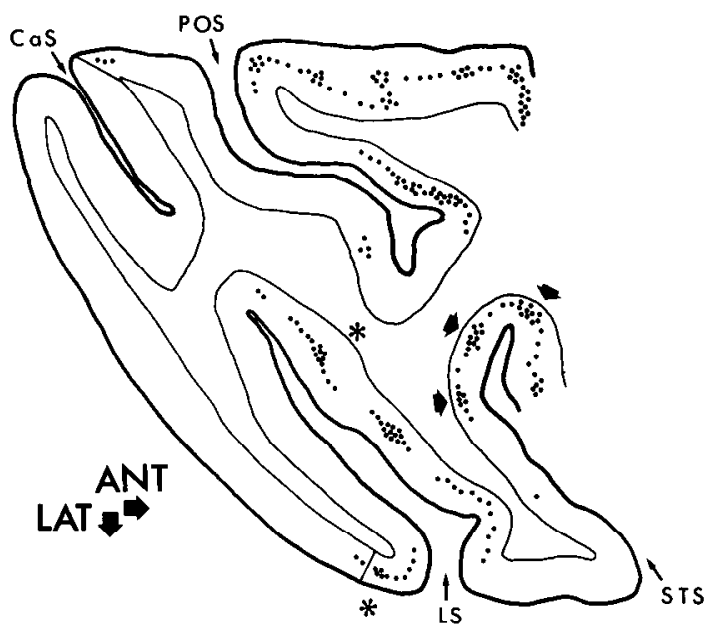

HRP
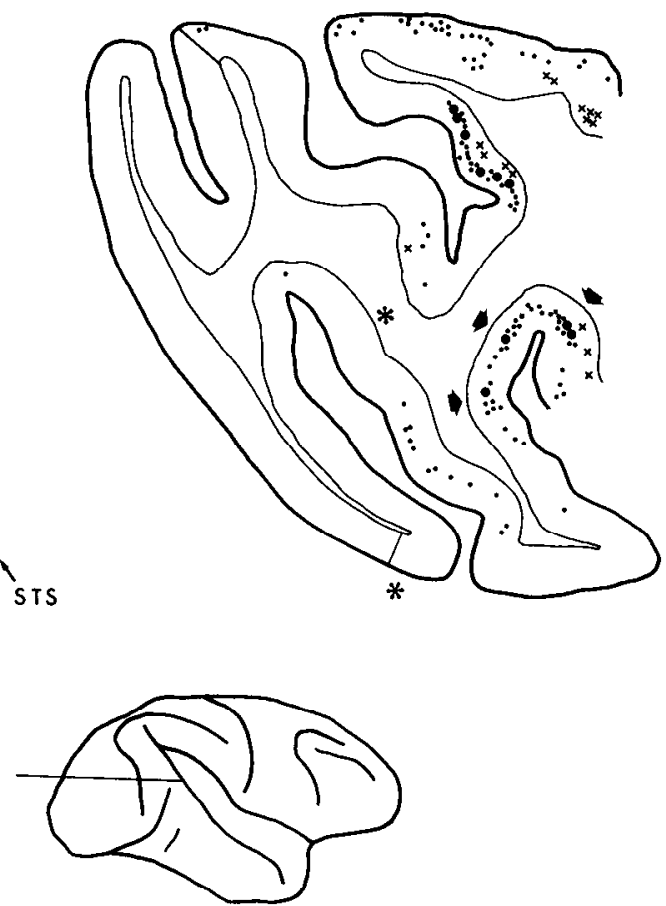

Figure 9. Comparison of callosal inputs and outputs. After callosal transection, adjacent horizontal sections taken at the level indicated in the hemisphere drawing were stained for degeneration (left) or reacted for HRP (right). On the right, crosses indicate cells below layer IV, small dots represent individual cells above layer IV, and large dots represent clusters of five cells. With the exception of the two sites denoted by asterisks, there are marked similarities in the distribution of callosal inputs and outputs, especially in the medial part of the STS (arrows). $A N T$, anterior; $L A T$, lateral. 

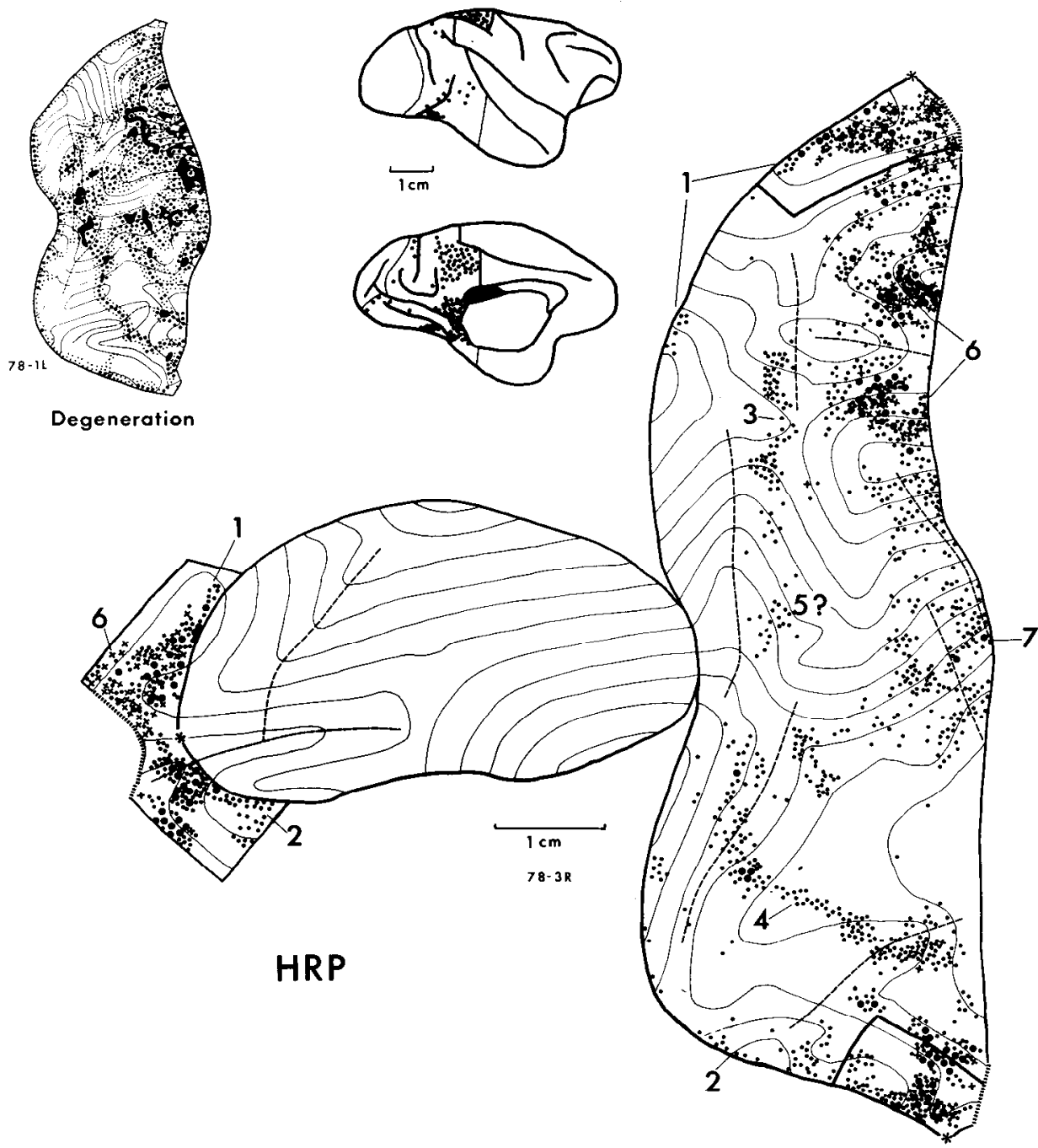

Figure 10. Regional distribution of callosal projection cells. The same symbols as in the preceding figure were used. There are obvious similarities with the pattern of callosal fiber terminations (inset, upper left); zones corresponding to previously identified callosal recipient zones are indicated on the map. Extrastriate cortex in the vicinity of the anterior calcarine sulcus ( solid outline) is represented twice on the map.

of origin of callosal and anterior commissure projections from parts of the superior temporal sulcus, inferior temporal gyrus, and parahippocampal gyrus. In view of the known degree of individual variability of callosal connections, though, firm conclusions on this issue should not be drawn without further experimentation, preferably by tracing both pathways in the same animal.

Laminar distribution of cells. An important feature of the callosal projection pattern shown in Figures 9 and 10 is that there are marked regional variations in the ratio of cells labeled in deep versus superficial layers. Along the V1/V2 border, there were no deep layer callosal projection neurons in any of the hemispheres that we examined except in a circumscribed region in the anterior calcarine sulcus (see below). Occasional labeled cells in deep layers (crosses in Figs. 9 and 10) were found in the rest of the occipital and temporal lobes, including the regions containing visual areas $\mathrm{V} 3, \mathrm{~V} 3 \mathrm{~A}, \mathrm{~V} 4, \mathrm{VP}$, and MT, but they never constituted more than $10 \%$ of the total population of labeled cells in these areas. The highest incidence of labeling in deep layers was in the parietal lobe, including both banks of the intraparietal sulcus and the medial wall of the hemisphere. Throughout this region, $10 \%$ to $50 \%$ of labeled cells were in deep layers (crosses), with the proportions in layers 5 and 6 being roughly similar. Interestingly, the high incidence of deep layer callosal projection occurs only in cortex anterior to known topographically organized visual areas. However, we do not know yet how closely the transitions in the two types of organization correspond.

One region of special interest in which there was extensive retrograde labeling in Figure 10 was the vicinity of the prostriate area in the anterior calcarine sulcus. As illustrated previously (Fig. 4), the optimal representation of this region is one in which the continuity between striate and extrastriate cortex is preserved. For this region, the relevant portion of extrastriate cortex has been represented twice in Figure 10, once in the standard fashion at the extreme top and bottom of the map and again on the far left adjoining striate cortex. The distri- 
bution of labeled cells in this region is remarkably similar to the pattern of callosal recipient cortex shown in Figure 4. In particular, the strip of callosal projection cells along the V1/V2 border is interrupted precisely in the region where the prostriate area adjoins V1 (i.e., adjacent to the far periphery representation in V1). In addition, the strips above and below the gap widen and merge with strips of callosal projection cells that lie outside of $\mathrm{V} 2$. It is also notable that the dorsoventral asymmetry in the laminar distribution of callosal projection cells is most striking in this region, as there is a sharp decrease in the incidence of deep layer callosal projection cells that occurs at the narrow neck between dorsal and ventral subdivisions of extrastriate cortex. It will be interesting to determine whether this reflects a significant asymmetry in the organization of visual areas adjoining the far peripheral representations in $\mathrm{V} 2$.

The V1/V2 border. We did not encounter any callosal projection cells at all inside striate cortex, even though their density was often quite high in V2, just across the sharply defined architectonic border. Correlated with this is an equally sharp transition in the density of callosal fiber terminations at the V1/V2 border, although sparse inputs (always much less than those in V2) often extend up to a millimeter or so into V1. Interestingly, the pattern is significantly different in most nonprimate species and in the bushbaby, a prosimian, where callosal projections both originate and terminate in a roughly symmetrical distribution across the V1/V2 border (Shatz, 1977; Towns et al., 1977; Swadlow et al., 1978; Weyand and Swadlow, 1980; Newsome and Allman, 1980; Cusick and Lund, 1981; but see Gould and Ebner, 1978).

\section{Discussion}

Although there have been numerous studies of interhemispheric connections in macaque visual cortex, many importanl features of this pathway have remained poorly understood. The major reason for this is obvious: the callosal inputs form a pattern which is both intrinsically complex and distributed over a highly convoluted region of cortex. The use of two-dimensional cortical maps obviates many of the difficulties associated with cortical convolutions and offers a convenient format for displaying this pattern accurately and with high resolution. Cortical maps clearly provide for a major economy of space. In the examples that we have shown, the information from 50 to 60 sections taken at $0.5-\mathrm{mm}$ intervals is displayed accurately in a tenth of the space that would be needed to show the individual sections at the same scale. More important, though, is the advantage of being able to appreciate a variety of spatial and topological relationships at a glance rather than having to rely on painstaking examination of large numbers of sections. It is instructive to note, for instance, that the dorsoventral asymmetry in callosal inputs to the occipital lobe, which is a prominent feature on the cortical maps and reflects an important asymmetry in the organization of visual areas, evidently escaped the attention of previous investigators. The asymmetry can be discerned from inspection of the sections illustrated by Myers (1962), but his original study did not comment on this point. A later review (Myers, 1965) explicitly suggested that callosal inputs to dorsal and ventral halves of the occipital lobe are arranged in mirror-symmetric fashion. Subsequent investigations either agreed with this suggestion (Zeki, 1970) or did not specifically address the issue (Cragg, 1969; Karol and Pandya, 1971).

The importance of having an accurate and detailed map of interhemispheric connections derives mainly from the usefulness of the pattern as an indication of vertical meridian representations, which, in turn, reflect the boundaries between certain visual areas. Callosal projections do not provide an ideal marker for either topographic organization or areal boundaries, however, and the limitations as well as strengths of the approach deserve careful scrutiny.

Callosal inputs and areal boundaries. We have already discussed the evidence that the pattern of callosal projections provides useful information concerning the location of five extrastriate visual areas, V2, V3, V3A, V4, and VP, in the macaque. The accuracy with which borders running along callosal recipient zones can be determined varies with the width of the zone and ranges from 1 to $2 \mathrm{~mm}$ for VP to 2 to $5 \mathrm{~mm}$ for V4. (The accuracy is even better for V2, but of course, its border with V1 can always be determined precisely even in Nissl-stained sections.) Although this degree of accuracy is far from ideal, it offers a marked improvement over what is attainable in the absence of information about callosal inputs. The individual variability that we found in callosal projection patterns suggests uncertainties ranging from several millimeters up to a centimeter or more for border estimates based solely on geographical landmarks. This estimate of variability is substantiated by the finding that the architectonically identifiable borders of MT vary by several millimeters with respect to landmarks within the superior temporal sulcus (Van Essen et al., 1981).

The usefulness of callosal projection patterns for discerning the organization of extrastriate visual cortex has been established in several other species besides the macaque. Callosal recipient zones related to areal boundaries have been demonstrated for V2, MT, VP, and DM (dorsomedial area) in the owl monkey (Newsome and Allman, 1980), areas 17, 18, and 19 and the lateral suprasylvian areas in the cat (Ebner and Myers, 1965; Hubel and Wiesel, 1965; Sanides, 1978), and for the V1/V2 boundary in the rabbit, rat, mouse, and hedgehog (Yorke and Caviness, 1975; Towns et al., 1977; Gould and Ebner, 1978; Cusick and Lund, 1981). Presumably, further studies in these and other species will reveal additional correlations, but it should not be expected that every topographically organized visual area will turn out to have a well defined callosal recipient strip concentrated along its perimeter. Indeed, this is already known not to be the case for MT in the macaque (Van Essen et al., 1981) and the dorsolateral (DL) and dorsointermediate (DI) areas in the owl monkey (Newsome and Allman, 1980).

The fine structure of callosal connections. One of the intriguing features of the callosal pathway is the intricate fine structure of the major callosal recipient zones. The degree of internal complexity varies considerably in different zones, with the simpler patterns showing relatively modest fluctuations in the overall width and density of 
callosal inputs and the more complex patterns showing irregular islands and anastamosing bands of moderate or high density inputs separated by regions containing little or no callosal input. Several general points about the fine structure of callosal connections are worth noting. First, complexities in fine structure are a widespread, perhaps universal feature of the interhemispheric communication system, having been described in both sensory and nonsensory regions of a variety of primate and nonprimate species (Ebner and Myers, 1965; Kunzle, 1976; Wise and Jones, 1976; Sanides, 1978; Imig and Brugge, 1978; Cipolloni and Peters, 1979; Newsome and Allman, 1980; Cusick and Lund, 1981). Second, the fine structure of callosal fiber terminations is usually mirrored by a similar distribution of callosal projection neurons, as shown in the present study for primate visual cortex and elsewhere for other sensory regions (Wise and Jones, 1976; Jones et al., 1979; but see Kelly and Wong, 1981). These similarities in callosal input and output patterns strengthen the suspicion that the fine structure of callosal connections somehow reflects important features of cortical organization. Third, in the macaque, we found considerable individual variability in fine structure (see also Van Essen and Zeki, 1978), and similar observations have been made in the cat and the rat (Sanides, 1978; Cusick and Lund, 1981). It seems reasonable, therefore, to regard every hemisphere as having a unique "callosal fingerprint." It will be interesting to determine whether opposite hemispheres from the same individual show less variability in their callosal fingerprints than is found between individuals.

The functional significance of the fine structure of callosal connections is an intriguing, but largely unresolved puzzle. One interesting clue is that "patchy" projections now are recognized to be a common organizational feature of many intrahemispheric cortical pathways (Zeki, 1971, 1978a; Jones and Wise, 1977; WongRiley, 1979; Jones et al., 1978; Rockland and Pandya, 1979; Montero, 1980; Imig and Reale, 1980; Van Essen et al., 1981). The dimensions of the patches and the spacing between them is variable, ranging from a fraction of a millimeter to several millimeters, which is similar to the range found for patches and bands associated with callosal projections. This suggests that the fine structure of callosal connections is not a manifestation of features specific to the interhemispheric communication pathway but, instead, may reflect aspects of internal organization common to both callosal free and callosal recipient cortex. This notion is supported by the evidence reported in the present study and by Zeki (1978a) that irregularities in inter- and intrahemispheric connections are superimposable when both patterns are mapped in the same hemisphere. It is still not obvious just what functional significance should be attributed to patchy cortical connections, but at least this insight serves to expand the scope of experimental analyses which can be brought to bear on the issue.

One possibility which has already been mentioned is that the fine structure of callosal connections to extrastriate visual cortex may be related to irregularitios in topographic organization, with the density of callosal connections at different sites reflecting the extent to which receptive fields extend into the ipsilateral hemifield. Another possibility is that some cortical cells may receive inputs which arise from a large portion of the visual field but whose sole effect is to modulate the responsiveness to stimulation of the cell's conventionally defined receptive field. If some of these modulatory influences were mediated by projections from the opposite hemisphere and if the extent of such influences were not uniform within the cortex, then they could contribute to the complexity of callosal connections without manifesting themselves as obvious irregularities in topographic organization. A third possibility is that some callosal connections may mediate nonvisual influences relating, for example, to eye movements or visual attention. Lastly, it is well established that the corpus callosum serves as a conduit for the interhemispheric transfer of pattern discrimination capacities (see Hamilton, 1982). The nature of the information actually transmitted between hemispheres in these situations is not known, and it may be that some of the information transfer is of a type qualitatively different from any of the possibilities just outlined (e.g., having to do with memories or strategies). None of these possibilities are mutually exclusive, and ascertaining their relative importance will be a difficult task. Obviously, any particular factor need not have the same importance in all regions of cortex; therefore, it is important to use caution in generalizing from the fragmentary information available on the issue. Nonetheless, enough is known to warrant brief discussion of several points.

(i) Direct comparisons between visual topography and the fine structure of callosal inputs in the same hemisphere have shown significant correlations in areas V3A and V4 of the macaque (Van Essen and Zeki, 1978) and at the boundary between areas 18 and 19 in the cat (Sanides and Albus, 1980). Also, the close relationship described above between irregularities in callosal projections and the patchiness of ipsilateral projections from sites of vertical meridian representation is at least consistent with both patterns reflecting complexities in topographic organization. In order to estimate the degree to which complexities in visual topography can account for the fine structure of callosal inputs, it will be important to have more quantitative analyses of local variations in the density of callosal inputs coupled with more detailed descriptions of irregularities in topographic organization.

(ii) Several studies involving focal lesions or tracer injections in individual extrastriate areas have demonstrated projections to the "homotopic" or corresponding portion of the same area in the opposite hemisphere (Spatz and Tigges, 1972; Wagor et al., 1975; Segraves and Rosenquist, 1982). This is a puzzling arrangement insofar as the connections are apparently between sites disposed mirror symmetrically across the vertical meridian representation. Since cells with highly elongated or dumbbellshaped receptive fields have not been described in these areas, it seems more plausible to suppose that this component of the callosal pathway mediates some kind of modulatory influence. A precedent for such modulatory interactions has been described in cat visual cortex (Rizzolatti and Camarda, 1977).

(iii) Behavioral enhancement of visual responsiveness 
has been demonstrated in several extrastriate visual areas in the macaque (Robinson et al., 1978, 1980; Fischer et al., 1981). There is no direct evidence implicating the corpus callosum in the mediation of such interactions, but it is worth noting that the enhancement effects are particularly pronounced in regions such as posterior parietal cortex, where callosal inputs are both extensive and complex.

Regional distribution of callosal projection cells. Since the advent of techniques for using HRP as a retrograde tracer in the CNS, there have been numerous studies of the cells of origin of the callosal pathway in various mammalian species. Previous studies in the macaque have shown callosal projections originating from cells at the V1/V2 border as well as in other regions of extrastriate visual cortex (Winfield et al., 1975; Lund et al., 1976; Rockland and Pandya, 1979), but the present study is the first to map their spatial distribution in detail. Not surprisingiy, we found that the distribution of callosal projection cells is very similar to that of callosal fiber terminations and thus reflects topographic organization to the same degree in various regions of cortex. The major dissinilarily in callosal inputs versus outputs is in inferotemporal cortex: this region receives inputs via the corpus callosum and, in its anterior portion, via the anterior commissure (Zeki, 1973), but its projections to the contralateral hemisphere pass predominantly through the anterior commissure and not the corpus callosum (Jouandet and Gazzaniga, 1979; Hamilton, 1982; see Fig. 10).

Although callosal fibers originate and terminate in similar overall patterns within extrastriate cortex, this does not imply that the connections are strictly homotopic (i.e., that any one locus projects only to the mirrorsymmetric locus in the opposite hemisphere). To the contrary, many callosal projections have been shown to be heterotopic (Zeki, 1971; Hedreen and Yin, 1981; Segraves and Rosenquist, 1982), and it has even been demonstrated that there are reciprocal interhemispheric connections between cortical and subcortical structures such as the claustrum (LeVay and Sherk, 1981).

Laminar distribution of callosal inputs and outputs. One of the basic principles that has emerged over the past decade from neuroanatomical studies of the cerebral cortex is that the various cortical layers each have highly distinctive patterns of inputs and outputs. It is instructive, therefore, to compare the laminar organization of callosal connections with that of other pathways, particularly intrahemispheric cortico-cortical connections.

In primates, it generally has been found that callosal inputs terminate predominantly in layer IV (e.g., Zeki, 1970; Karol and Pandya, 1971; Jones et al., 1979; Rockland and Pandya, 1979; Newsome and Allman, 1980). These studies also have demonstrated substantial inputs to other layers, primarily below layer IV in studies using degeneration stains (which are likely to overemphasize fibers of passage) and primarily above layer IV in autoradiographic studies (which are likely to give a more accurate picture). This pattern is quite similar to that of the major ipsilateral cortical pathways progressing outward from the primary sensory areas (Jones et al., 1978; Wong-Riley, 1979; Rockland and Pandya, 1979). In non- primates, the laminar distribution of cortico-cortical projections differs from that found in primates, but once again, the patterns for ipsilateral and contralateral projections are usually quite similar. Both inter- and intrahemispheric projections are densest to layer III, relatively sparse in layer IV, and in some regions, dense also in layer V or VI (Wise and Jones, 1976; Shatz, 1977; Imig and Brugge, 1978; Cipolloni and Peters, 1979; Imig and Reale, 1980; Kelly and Wong, 1981).

Similar comparisons can be made with regard to the cells of origin of inter- and intrahemispheric cortical pathways. In both primates and nonprimates, callosal projection cells are most numerous in layer III, but in all areas studied, there is a significant population in one or more additional layers (Jacobson and Trojanowski, 1974; Yorke and Caviness, 1975; Wise and Jones, 1976; Shatz, 1977; Swadlow et al., 1978; Jones et al., 1979; Kelly and Wong, 1981; present study). The predominance of callosal projection cells in layer III fits well with the evidence that this layer is generally the largest source of efferent projections to regions presumed to be at higher processing levels (Gilbert and Kelly, 1975; Jones et al., 1978; Rockland and Pandya, 1979). The significance of the deep layer callosal projection cells in various regions has not been firmly established, but one interesting possibility is that they are associated with feedback pathways from "higher" areas on one side to "lower" areas on the other. This suggestion is based on the finding that deep layer cells are a major source of intrahemispheric feedback pathways (e.g., Gilbert and Kelly, 1975; Wong-Riley, 1979; Jones et al., 1978; Rockland and Pandya, 1979). It is supported by the finding of a high incidence of retrogradely labeled cells in the deep layers of area 19 and lateral suprasylvian cortex after HRP injections either ipsilaterally in area 17 or area 18 or contralaterally at the 17/18 border (Gilbert and Kelly, 1975; Shatz, 1977). The absence in the macaque of both deep layer callosal projection cells at the V1/V2 border and substantial callosal inputs to V1 is also consistent with this suggestion. On the other hand, the finding of occasional deep layer callosal projection cells even in area 17 of the cat (Shatz, 1977 ) indicates that this is not an invariant rule.

Concluding remarks. Less than 2 decades ago, it was first realized that the corpus callosum could serve as a pathway linking cortical regions in opposite hemispheres that represent adjoining portions of a continuous sensory surface (Choudhury et al., 1965; see Berlucchi, 1972). Thus, the idea was generated that callosal connections provide for "perceptual unity" across the two halves of the visual field and the two halves of the body surface. Subsequent research has confirmed the importance of this notion and as well has emphasized that there are other fascinating aspects of callosal function such as those revealed by studies of higher cortical function (e.g., Gazzaniga, 1970).

One of the important insights which has emerged from the detailed analysis of cortical connections is the fundamental similarity between interhemispheric and intrahemispheric cortical pathways. Of course, both are genuine "cortico-cortical" pathways, but despite this, there is no way of knowing a priori how divergent their patterns of organization might be. Based on information 
gained over the past few years, it is evident that they have several important features in common. Both pathways originate from populations of cells having similar laminar distributions (predominantly in layer III), and they terminate in a remarkably similar fashion (layer IV and above in primates; mainly outside of layer IV in nonprimates). Both pathways show a striking degree of fine structure in spatial distribution parallel to the cortical surface, as evidenced by patchy and banded patterns of connections. To be sure, one should not expect the pathways to be identical in all respects. Nonetheless, it seems reasonable to regard the corpus callosum as providing essentially the same types of connections between appropriate regions in opposite hemispheres as are provided by local, ipsilateral cortical connections. By this hypothesis, discussed also by Hamilton (1982), the callosal pathway is not chiefly involved in carrying out "special" functions, at least in regions lacking hemispheric asymmetries. Rather, its most distinctive feature is simply its physical accessibility, which presumably will continue to make it well suited to assist in the exploration of the many diverse aspects of cortical function.

\section{References}

Allman, J. M., and J. H. Kaas (1974) A crescent-shaped visual area surrounding the middle temporal area (MT) in the owl monkey (Aotus trivirgatus). Brain Res. 81: 199-213.

Benevento, L. A., and F. F. Ebner (1971) The areas and layers of corticocortical terminations in the visual cortex of the Virginia opossum. J. Comp. Neurol. 141: 157-190.

Berlucchi, G. (1972) Anatomical and physiological aspects of visual functions of corpus callosum. Brain Res. 37: 371-392.

Choudhury, P. B., D. Whitteridge, and M. E. Wilson (1965) The function of the callosal connections of the visual cortex. Q. J. Exp. Physiol. 50: 214-219.

Cipolloni, P. B., and A. Peters (1979) The bilaminar and banded distribution of the callosal terminals in posterior neocortex of the rat. Brain Res. 176: 33-47.

Cowan, W. M., D. I. Gottlieb, A. E. Hendrickson, J. L. Price, and T. A. Woolsey (1972) The autoradiographic demonstration of axonal connections in the central nervous system. Brain Res. 37: 21-51.

Cragg, B. G. (1969) The topography of the afferent projections in the circumstriate visual cortex of the monkey studied by the Nauta method. Vision Res. 9: 733-747.

Cusick, C. G., and R. D. Lund (1981) The distribution of the callosal projection to the occipital visual cortex in rats and mice. Brain Res. 214: 239-259.

Daniel, P. M., and D. Whitteridge (1961) The representation of the visual field on the cerebral cortex in monkeys. J. Physiol. (Lond.) 159: 203-221.

Desimone, R., and C. G. Gross (1979) Visual areas in the lemporal corlex of the macaque. Brain Res. 178: 363-380.

Desimone, R., J. Fleming, and C. G. Gross (1980) Prestriate afferents to inferior temporal cortex: An HRP study. Brain Res. 184: 41-55.

Ebner, F. F., and R. E. Myers (1965) Distribution of corpus callosum and anterior commissure in cat and raccoon. $\mathrm{J}$. Comp. Neurol. 124: 353-366.

Fischer, B., R. Boch, and M. Bach (1981) Stimulus versus eye movements: Comparison of neural activity in the striate and prelunate visual cortex (A 17 and A 19) of trained rhesus monkey. Exp. Brain Res. 43: 69-77.

Gallyas, F. (1979) Silver staining of myelin by means of physical development. Neurol. Res. 1: 203-209.
Gazzaniga, M. S. (1970) The Bisected Brain, Appleton-Century-Crofts, New York.

Gilbert, C. D., and J. P. Kelly (1975) 'The projections of cells in different layers of the cat's visual cortex. J. Comp. Neurol. 163: 81-106.

Gould, H. J., and F. F. Ebner (1978) Connections of the visual cortex in the hedgehog (Paraechinus hypomelas). II. Corticocortical projections. J. Comp. Neurol. 177: 473-502.

Graham, R. C., Jr., and M. J. Karnovsky (1966) The early stages of absorption of injected horseradish peroxidase in the proximal tubules of mouse kidney: Ultrastructural cytochemistry by a new technique. J. Histochem. Cytochem. 14: 291302.

Gross, C. G., C. E. Rocha-Miranda, and D. B. Bender (1972) Visual properties of neurons in inferotemporal cortex of the macaque. J. Neurophysiol. 35: 96-111.

Hamilton, C. R. (1982) Mechanisms of interocular equivalence. In Advances in the Analysis of Visual Behavior, D. Ingle, M. Goodale, and R. Mansfield, eds., pp. 693-717, MIT Press, Cambridge, MA.

Hedreen, J., and T. C. T. Yin (1981) Homotopic and heterotopic callosal afferents of caudal inferior parietal lobule in $\mathrm{Macaca}$ mulatta. J. Comp. Neurol. 197: 605-621.

Hubel, D. H., and T. N. Wiesel (1965) Receptive fields and functional architecture in two non-striate visual areas (18 and 19) of the cat. J. Neurophysiol. 28: 229-289.

Imig, T. J., and J. F. Brugge (1978) Sources and terminations of callosal axons related to binaural and frequency maps in primary auditory cortex of the cat. J. Comp. Neurol. 182: $637-660$.

Imig, T. J., and R. A. Reale (1980) Patterns of cortico-cortical connections related to tonotopic maps in cat auditory cortex. J. Comp. Neurol. 192: 293-332.

Jacobson, S., and J. Q. Trojanowski (1974) The cells of origin of the corpus callosum in rat, cat and rhesus monkey. Brain Res. 74: 149-155.

Jones, E. G., and S. P. Wise (1977) Size, laminar and columnar distribution of efferent cells in the sensory-motor cortex of monkeys. J. Comp. Neurol. 175: 391-438.

Jones, E. G., J. D. Coulter, and S. H. C. Hendry (1978) Intracortical connectivity of architectonic fields in the somatic sensory, motor and parietal cortex of monkeys. J. Comp. Neurol. 181: 291-348.

Jones, E. G., J. D. Coulter, and S. P. Wise (1979) Commissural columns in the sensory-motor cortex of monkeys. J. Comp. Neurol. 188: 113-136.

Jouandet, M. L., and M. S. Gazzaniga (1979) Cortical field of origin of the anterior commissure of the rhesus monkey. Exp. Neurol. 66: 381-397.

Karol, E. A., and D. N. Pandya (1971) The distribution of the corpus callosum in the rhesus monkey. Brain 94: 471-486.

Kelly, J. P., and D. Wong (1981) Laminar connections of the cat's auditory cortex. Rrain Res. 212: 1-15.

Kunzle, H. (1976) Alternating afferent zones of high and low axon terminal density within the macaque motor cortex. Brain Res. 106: 365-370.

LeVay, S., and H. Sherk (1981) The visual claustrum of the cat. I. Structure and connections. J. Neurosci. 1: 956-980.

Lund, J. S., R. D. Lund, A. E. Hendrickson, A. H. Bunt, and A. F. Fuchs (1976) The origin of efferent pathways from the primary visual cortex, area 17 , of the macaque monkey as shown by retrograde transport of horseradish peroxidase. $J$. Comp. Neurol. 164: 287-304.

Lynch, J. C., V. B. Mountcastle, W. H. Talbot, and T. C. T. Yin (1977) Parietal lobe mechanisms for directed visual attention. J. Neurophysiol. 40: 362-389.

Montero, V. M. (1980) Patterns of connections from the striate cortex to cortical visual areas in superior temporal sulcus of 
macaque and middle temporal gyrus of owl monkey. J. Comp. Neurol. 189: 45-59.

Motter, B. C., and V. B. Mountcastle (1981) The functional properties of the light-sensitive neurons of the posterior parietal cortex studied in waking monkeys: Foveal sparing and opponent vector organization. J. Neurosci. 1: 3-26.

Myers, R. E. (1962) Commissural connections between occipital lobes of the monkey. J. Comp. Neurol. 118: 1-16.

Myers, R. E. (1965) Organization of visual pathways. (General discussion). In Functions of the Corpus Callosum, E. G. Ettlinger, ed., pp. 133-138, Little, Brown and Co., Boston.

Newsome, W. T., and J. M. Allman (1980) Interhemispheric connections of visual cortex in the owl monkey, Aotus trivirgatus, and the bushbaby, Galago senegalensis. J. Comp. Neurol. 194: 209-233.

Newsome, W. T., J. H. R. Maunsell, and D. C. Van Essen (1980) Areal boundaries and topographic organization of the ventral posterior area (VP) of the macaque monkey. Soc. Neurosci. Abstr. 6: 579 .

Pandya, D. N., E. A. Karol, and D. Heilbronn (1971) The topographical distribution of interhemispheric projections in the corpus callosum of the rhesus monkey. Brain Res. 32: $31-$ 43.

Rizzolatti, G., and R. Camarda (1977) Influence of the presentation of remote visual stimuli on visual responses of cat area 17 and lateral suprasylvian area. Exp. Brain Res. 29: 107-122.

Robinson, D. L., M. E. Goldberg, and G. B. Stanton (1978) Parietal association cortex in the primate: Sensory mechanisms and behavioral modulations. J. Neurophysiol. 41: 910 932.

Robinson, D. L., J. S. Baizer, and B. M. Dow (1980) Behavioral enhancement of visual responses of prestriate neurons of the rhesus monkey. Invest. Ophthalmol. Vis. Sci. 19: 1120-1123.

Rockland, K. S., and D. N. Pandya (1979) Laminar origins and terminations of cortical connections of the occipital lobe in the rhesus monkey. Brain Res. 179: 3-20.

Sanides, D. (1978) The retinotopic distribution of visual callosal projections in the suprasylvian visual areas compared to the classical visual areas $(17,18,19)$ in the cat. Exp. Brain Res. 33: 435-443.

Sanides, D., and K. Albus (1980) The distribution of interhemispheric projections in area 18 of the cat: Coincidence with discontinuities of the representation of the visual field in the second visual area (V2). Exp. Brain Res. 38: 237-240.

Sanides, F. (1970) Functional architecture of motor and sensory cortices in primates in the light of a new concept of neocortex evolution. In The Primate Brain, C. R. Noback and W. Montagna, eds., pp. 137-208, Appleton-Century-Crofts, New York.

Schein, S. J., R. T. Marrocco, and F. M. de Monasterio (1982) Is there a high concentration of color-selective cells in the V4 area of monkey visual cortex? J. Neurophysiol., in press.

Segraves, M. A., and A. C. Rosenquist (1982) The afferent and efferent callosal connections of retinotopically defined areas in cat cortex. J. Neurosci. 2: in press.

Seltzer, B., and D. N. Pandya (1978) Afferent cortical connections and architectonics of the superior temporal sulcus and surrounding cortex in the rhesus monkey. Brain Res. 149: 124.

Seltzer, B., and D. N. Pandya (1980) Converging visual and somatic sensory cortical input to the intraparietal sulcus of rhesus monkey. Brain Res. 192: 339-351.

Shatz, C. .J. (1977) Anatomy of interhemispheric connections in the visual system of Boston Siamese and ordinary cats. J. Comp. Neurol. 173: 497-518.

Spatz, W. B., and J. Tigges (1972) Experimental-anatomical studies on the "Middle Temporal Visual Area (MT)" in primates. I. Efferent cortico-cortical connections in the marmoset Callithrix jacchus. J. Comp. Neurol. 146: 451-464.
Swadlow, H. A., T. G. Weyand, and S. G. Waxman (1978) The cells of origin of the corpus callosum in rabbit visual cortex. Brain Res. 156: 129-134.

Towns, L. C., R. A. Giolli, and D. A. Haste (1977) Corticocortical fiber connections of the rabbit visual cortex: A fiber degeneration study. J. Comp. Neurol, 173: 537-560.

Ungerleider, L. G., and M. Mishkin (1979) The striate projection zone in the superior temporal sulcus of Macaca mulatta: Location and topographic organization. J. Comp. Neurol. 188: $347-366$.

Van Essen, D. C. (1979) Visual areas of the mammalian cerebral cortex. Annu. Rev. Neurosci. 2: 227-263.

Van Essen, D. C., and J. L. Bixby (1978) The distribution of cells projecting interhemispherically in extrastriate visual cortex of the macaque. Soc. Neurosci. Abstr. 4: 649.

Van Essen, D. C., and J. H. R. Maunsell (1980) Two-dimensional maps of the cerebral cortex. J. Comp. Neurol. 191: 255-281.

Van Essen, D. C., and S. M. Zeki (1978) The topographic organization of rhesus monkey prestriate cortex. J. Physiol. (Lond.) 277: 193-226.

Van Essen, D. C., J. H. R. Maunsell, and J. L. Bixby (1979) Areal boundaries and topographic organization of visual areas V2 and V3 in the macaque monkey. Soc. Neurosci. Abstr. 5: 812.

Van Essen, D. C., J. H. R. Maunsell, and J. L. Bixby (1981) The middle temporal visual area in the macaque: Myeloarchitecture, connections, functional properties and topographic organization. J. Comp. Neurol. 199: 293-326.

Wagor, E., C. S. Lin, and J. H. Kaas (1975) Some cortical projections of the dorsomedial visual area (DM) of association cortex in the owl monkey, Aotus trivirgatus. J. Comp. Neurol. 163: 227-250.

Weyand, T. G., and H. A. Swadlow (1980) Interhemispheric striate projections in the prosimian primate, Galago senegalensis. Brain Behav. Evol. 17: 473-477.

Wiitanen, J. T. (1969) Selective silver impregnation of degenerating axons and axon terminals in the central nervous system of the monkey (Macaca mulatta). Brain Res. 14: $546-548$.

Winfield, D. A., K. C. Gatter, and T. P. S. Powell (1975) Certain connections of the visual cortex of the monkey shown by the use of horseradish peroxidase. Brain Res. 92: 456-461.

Wise, S. P., and E. G. Jones (1976) The organization and postnatal development of the commissural projection of the rat somatic sensory cortex. J. Comp. Neurol. 168: 313-344.

Wong-Riley, M. (1979) Columnar cortico-cortical interconnections within the visual syslem of the squirrel and macaque monkeys. Brain Res. 162: 201-217.

Yorke, C. H., Jr., and V. S. Caviness, Jr. (1975) Interhemispheric neocortical connections of the corpus callosum in the normal mouse: A study based on anterograde and retrograde methods. J. Comp. Neurol. 164: 233-246.

Zeki, S. M. (1969) Representation of central visual fields in prestriate cortex of monkey. Brain Res. 14: 271-291.

Zeki, S. M. (1970) Interhemispheric connections of prestriate cortex in monkey. Brain Res. 19: 63-75.

Zeki, S. M. (1971) Cortical projections from two prestriate areas in the monkey. Brain Res. 34: 19-35.

Zeki, S. M. (1973) Comparison of the cortical degeneration in the visual regions of the temporal lobe of the monkey following section of the anterior commissure and the splenium. J. Comp. Neurol. 148: 167-176.

Zeki, S. M. (1977) Colour coding in the superior temporal sulcus of rhesus monkey visual cortex. Proc. R. Soc. Lond. (Biol.) 197: 195-223.

Zeki, S. M. (1978a) The cortical projections of foveal striate cortex in the rhesus monkey. I. Physiol. (Lond.) 277: 227244. 
Zeki, S. M. (1978b) The third visual complex of rhesus monkey prestriate cortex. J. Physiol. (Lond.) 277: 245-272.

Zeki, S. M. (1978c) Uniformity and diversity of structure and function in rhesus monkey prestriate visual cortex. J. Physiol. (Lond.) 277: 273-290.

Zeki, S. M. (1978d) Functional specialisation in the visual cortex of the rhesus monkey. Nature 274: 423-428.

Zeki, S. M., and D. R. Sandeman (1976) Combined anatomical and electrophysiological studies on the boundary between the second and third visual areas of rhesus monkey cortex. Proc. R. s, sc. Lond. (Biol.) 194: 555-562. 